\title{
Electronic structure of mixed valent systems
}

\author{
V.N.Antonov ${ }^{1}$, A.P.Shpak ${ }^{1}$, A.N. Yaresko ${ }^{2}$ \\ 1 Institute of Metal Physics of the National Academy of Sciences of Ukraine, \\ 36 Vernadsky str., 03142 Kiev \\ 2 Max Planck Institute for Physics of Complex Systems, \\ D-01187 Dresden, Germany
}

Received March 24, 2004

The electronic structure of $\mathrm{Tm}$ and $\mathrm{Sm}$ monochalcogenides, $\mathrm{SmB}_{6}$ and $\mathrm{Yb}_{4} \mathrm{As}_{3}$ is theoretically investigated from the first principles, using the fully relativistic Dirac LMTO band structure method. The electronic structure is obtained using the local spin-density approximation (LSDA), as well as the so-called LSDA $+U$ approach. While the standard LSDA approach is incapable of correctly describing the electronic structure of such materials due to the strong on-site Coulomb repulsion, the $\mathrm{LSDA}+U$ approach is remarkably accurate in providing a detailed agreement with experiment for a number of properties.

Key words: strongly correlated systems, optics, band structure

PACS: $71.28 .+d, 71.25 . P i, 75.30 . M b$

\section{Introduction}

Determination of the energy band structure of solids is a many-body problem. Band theory, i.e., a mean-field theory to treat this problem, in the framework of the local spin density approximation (LSDA), has been successful for many kinds of materials, and has become the de facto tool of first-principle calculations in solid state physics. It has significantly contributed to the understanding of material properties at the microscopic level. However, there are some systematic errors which have been observed when using the LSDA. In particular, the LSDA fails to describe the electronic structure and properties of $f$ electron systems in which the interaction among the electrons is strong. These are now called strongly correlated electron systems and many new concepts to address these phenomena have been constructed. However, the understanding of these systems is incomplete. A wide variety of physical properties arise from the correlations among $f$ electrons: metal-insulator transitions, valence fluctuations in the Kondo effect, heavy fermion behavior, superconductivity, 
and so on.

With the aim of undertaking a systematic investigation of the trends in some mixed valent $4 f$ compounds we present the theoretically calculated electronic structure, optical and photo-emission spectra for the Tm and Sm monochalcogenides, $\mathrm{SmB}_{6}$ and $\mathrm{Yb}_{4} \mathrm{As}_{3}$ compounds.

The paper is organized as follows. Section 2 presents a brief description of the computational details. Section 3 is devoted to the electronic structure and optical and photo-emission spectra of $\operatorname{TmX}(\mathrm{X}=\mathrm{S}, \mathrm{Se}, \mathrm{Te}), \mathrm{SmS}(\mathrm{S}=\mathrm{S}, \mathrm{Se}, \mathrm{Te}), \mathrm{SmB}_{6}$, and $\mathrm{Yb}_{4} \mathrm{As}_{3}$ calculated in the LSDA and LSDA $+U$ approximations. The theoretical calculations are compared to the experimental measurements. Finally, the results are summarized in section 4 .

\section{Computational details}

The application of plain LSDA calculations to $f$-electron systems is often inappropriate due to the correlated nature of the $f$ shell. There have actually been different attempts to improve the LSDA in order to treat correlated electrons: $G W$ approach, Dynamical Mean Field Theory, Time-dependent Density Functional Theory, Self-interaction Correction, and LSDA $+U$ method being the most frequently used ones $[1,2]$. To better account for the on-site $f$-electron correlations, we have adopted the LSDA $+U$ approach $[3,4]$ as a suitable model.

A rigorous formulation for the quasiparticle properties of solids is the Green function approach. The self-energy $\Sigma=G_{0}^{-1}-G^{-1}$ of the single-particle Green function $G$ is energy dependent and yields the correlation corrections to the single-particle (mean-field) approximation to the quasi-particle excitation spectrum described by $G_{0}$. With a number of plausible assumptions, the LSDA $+U$ approach has been related to the so-called GW approximation to $\Sigma$ in [5]. Already the simplest random phase approximation applied to $\Sigma$ for the Hubbard model yields a jump of $\Sigma(\epsilon)$ at the Fermi level $\epsilon_{\mathrm{F}}$ by the Hubbard $U$. The more elaborate analysis of [5] results in a correlation correction to the mean-field approximation of the self-energy, which is $U_{\text {eff }} / 2$ downward below the Fermi level and $U_{\text {eff }} / 2$ upward above the Fermi level. Since the mean field theory in a crystal always describes a delocalized situation and the LSDA Kohn-Sham potential is a well proved approximation to the self-energy of weakly correlated situations [6], the suggestion is

$$
\Sigma\left(\vec{r}, \vec{r}^{\prime} ; \epsilon\right) \approx \delta\left(\vec{r}-\vec{r}^{\prime}\right) v_{\mathrm{LSDA}}(\vec{r})+P_{m} \frac{U_{\mathrm{eff}}}{2}\left[\theta\left(\epsilon-\epsilon_{\mathrm{F}}\right)-\theta\left(\epsilon_{\mathrm{F}}-\epsilon\right)\right] P_{m},
$$

where $P_{m}$ is the projector onto a strongly correlated $m$ state.

The LSDA $+U$ approach simply uses (2.1) to replace the LSDA Kohn-Sham potential in the self-consistency loop. This can be considered as a rough approximation to $\Sigma$. Since the potential shift is taken to be constant in space, it does not deform the Kohn-Sham orbital $\phi_{m}$. However, it shifts the levels of strongly correlated motion away from the Fermi level and thus removes incorrect hybridization with conduction states, which would spoil the calculated ground state spin density. On the other 
hand, being also understood as an approximation to $\Sigma$, it hopefully yields for the Kohn-Sham band structure the same quality of a working approximation to the quasiparticle excitation spectrum as it does in the case of weakly correlated metals. Estimates for $U_{\text {eff }}$ may be obtained from the constrained density functional calculations or from GW calculations in which case the approach is parameter-free. Most reliable are those results which do not very sensitively depend on the actual value of $U_{\text {eff }}$ within a certain reasonable range.

The details of the computational method are described in our previous papers, $[7,8]$ and here we only mention several aspects. The calculations were performed using the LMTO method $[9,10]$ in the atomic sphere approximation (ASA) with the combined correction term taken into account. We used the von Barth-Hedin parametrization [11] for the exchange-correlation potential. Brillouin zone (BZ) integrations were performed using the improved tetrahedron method [12]. The combined correction terms were also included into the calculation of the optical matrix elements [13]. We calculated the absorptive part of the optical conductivity in a wide energy range and then the Kramers-Kronig transformation was used to calculate the dispersive parts of the optical conductivity from the absorptive parts.

We have adopted the LSDA $+U$ method as a different level of approximation to treat the electron-electron correlations. The LSDA $+U$ method has proved to be an efficient and reliable tool for calculating the electronic structure of systems where the Coulomb interaction is strong enough to cause localization of the electrons. It works not only for nearly core-like $4 f$ orbitals of rare-earth ions $[8,14-19]$, but also for such systems as transition metal oxides, where $3 d$ orbitals quite strongly hybridize with oxygen $2 p$ orbitals [20-23], and uranium compounds [7,24-30].

\section{Results and discussion}

\subsection{Tm monochalcogenides}

The Tm monochalcogenides TmS, TmSe and TmTe constitute a well known family of strongly correlated electron systems. They form a very interesting group of materials in which many characteristic phenomena are expressed. In the series of the Tm monochalcogenides one has an interesting possibility to go from integer trivalent metallic TmS to integral divalent semiconducting TmTe through intermediate valence TmSe [31-33]. The unique feature of thulium compounds compared with intermediate valence materials containing cerium, samarium or europium is that both ground-state configurations of thulium have a non-zero magnetic moment. TmS exhibits antiferromagnetic order which is an almost type II sinusoidally modulated structure below $T_{\mathrm{N}}=6.2 \mathrm{~K}$ [34]. It has been classified as a Kondo lattice compound due to a metallic resistivity which increases Kondo-like down to about $15 \mathrm{~K}$ [35-38]. TmSe has attracted interest because of the valence fluctuation between $4 f^{12}$ and $4 f^{13}$ configurations, Kondo effects, antiferromagnetic order, and a possible metal-insulator transition. The peculiarity of this compound is the involvement of two magnetic valence states $\mathrm{Tm}^{2+}$ and $\mathrm{Tm}^{3+}$ in the valence fluctuation. Evidence 
comes from photoemission experiments [31,32,39-43], as well as from the measurements related to magnetic properties, resistivity and specific heat [44-48]. However, the situation is so complicated that there is no overall consistent explanation of the physical properties of this compound. The resistivity of TmSe shows a Kondo-like logarithmic temperature dependence at high temperatures followed by a sharp increase at $T_{\mathrm{N}}=3.5 \mathrm{~K}$, which is thought to be a transition into an insulating state $[47,49,50]$. This anomaly in the vicinity of $T_{\mathrm{N}}$ shows a very complicated response to external magnetic fields and to pressure [46,51].

TmTe is a magnetic semiconductor with a localized $13^{\text {th }} f$ level between a filled Te- $5 p$ valence band and an empty Tm- $5 d$ conduction band [32]. The lattice constant and the Curie constant show that the Tm ions are divalent at an ambient pressure [31]. TmTe is interesting in its own right, especially since it was recently reported [52] to undergo a phase transition at $T_{\mathrm{Q}}=1.7 \mathrm{~K}$, far above the magnetic-ordering temperature $\left(T_{\mathrm{N}} \sim 0.2-0.4 \mathrm{~K}\right.$ depending on the specimen). This transition was ascribed to the onset of long-range ordering among the Tm quadrupolar moments, but the exact mechanism is still controversial [53].

\subsubsection{TmS}

Band structure The theoretical study of the electronic structure, optical and magneto-optical spectra of $\operatorname{TmX}(\mathrm{X}=\mathrm{S}$, Se and Te) has been done in [8]. There were performed three independent fully relativistic spin-polarized band structure calculations. The $4 f$ electrons have been considered as: (1) itinerant electrons using the local spin-density approximation; (2) fully localized, putting them in the core; and (3) partly localized using the LSDA $+U$ approximation. An important difference with respect to treating the $4 f$ electrons as core electrons is that in the LSDA $+U$ calculation all optical transitions from the $4 f$ states are taken into account.

Figure 1 shows the energy band structure of $\mathrm{TmS}$ for all three approximations. The energy band structure of TmS with the $4 f$ electrons in the core can be subdivided into three regions separated by energy gaps. The bands in the lowest region around $-15 \mathrm{eV}$ have mostly $\mathrm{S} s$ character with some amount of Tm $s p$ character mixed in. The next six energy bands are $\mathrm{S} p$ bands separated from the $s$ bands by an energy gap of about $6.5 \mathrm{eV}$. The width of the $\mathrm{S} p$ band is about $3.7 \mathrm{eV}$. The spin splitting of the $\mathrm{S} p$ bands is very small (about $0.06 \mathrm{eV}$ at the $\mathrm{X}$ symmetry point (figure 1)). The highest region can be characterized as Tm spin-split $d$ bands. It is important that the top of the $\mathrm{S} p$ bands is at $-3.5 \mathrm{eV}$ below the Fermi level since it means that all the interband transitions in the energy interval of 0.0 to $3.5 \mathrm{eV}$ participate within the Tm $d$ bands (see below). The sharp peaks in the DOS calculating in the LSDA at the Fermi energy and near $2 \mathrm{eV}$ below are due to $4 f_{7 / 2}$ and $4 f_{5 / 2}$ states respectively (figure 1 ).

The LSDA $+U$ band structure calculations [8] have been started from a $4 f^{12}$ configuration for the $\mathrm{Tm}^{3+}$ ion with twelve on-site $4 f$ energies shifted downward by $U_{\text {eff }} / 2$ and two levels shifted upwards by this amount. The energies of occupied and unoccupied $f$ levels are separated by approximately $U_{\text {eff }}$. We emphasize, however, that the $4 f$ states are not completely localized, but may hybridize, and their energy 

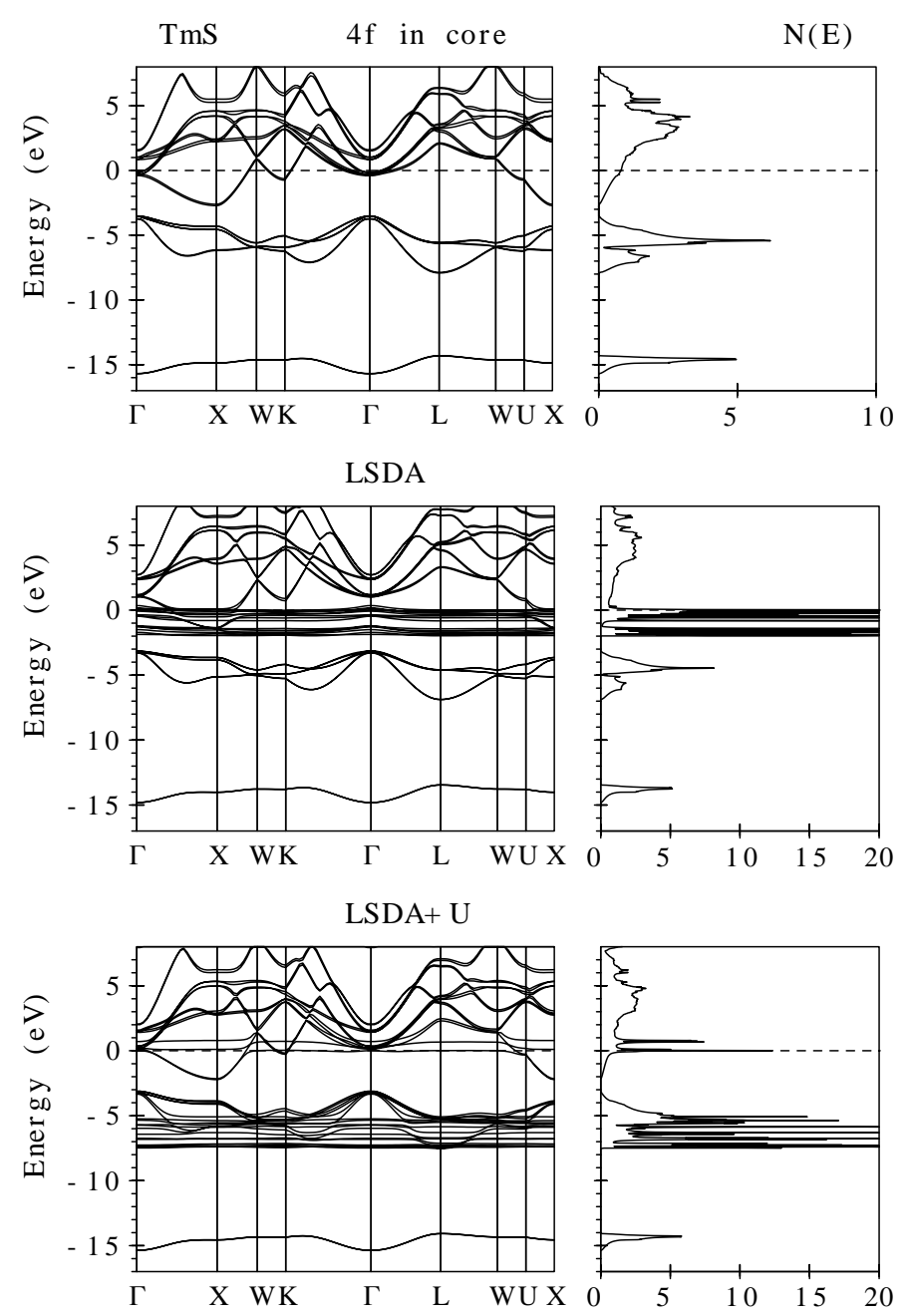

Figure 1. Self-consistent fully relativistic, spin-polarized energy band structure and total DOS (in states/(unit cell eV)) calculated for TmS [8] treating the $4 f$ states as: (1) fully localized ( $f f$ in core); (2) itinerant (LSDA); and (3) partly localized $(\mathrm{LSDA}+U)$.

positions together with all other states relax to self consistency.

The LSDA $+U$ energy bands and total density of states (DOS) of TmS for $U_{\text {eff }}=$ $6 \mathrm{eV}$ are shown in figure 1 . The Coulomb repulsion $U_{\text {eff }}$ strongly effects the electronic structure of TmS. For $\mathrm{Tm}^{3+}$ ions twelve $4 f$ bands are fully occupied and hybridize with $\mathrm{S} p$ states. The $14^{\text {th }} f$ hole level is completely unoccupied and well above the Fermi level. A hole $13^{\text {th }} f$ level is pinned at the Fermi level. Although there was used a starting configuration with zero occupation of $14^{\text {th }} f$ and $13^{\text {th }} f$ levels, in the process of self-consistent relaxation, the initially empty $13^{\text {th }} f$ level became partly occupied due to the pinning at the Fermi level with the occupation number equal to 0.12 (valence $2.88+$ ) in good agreement with the experimental estimations $(2.91+$ ) by off-resonance UPS [43]. A fundamental aspect of this observation is that the pinning of the $13^{\text {th }} f$ state at $E_{\mathrm{F}}$ turns out to be a robust property of the TmS 

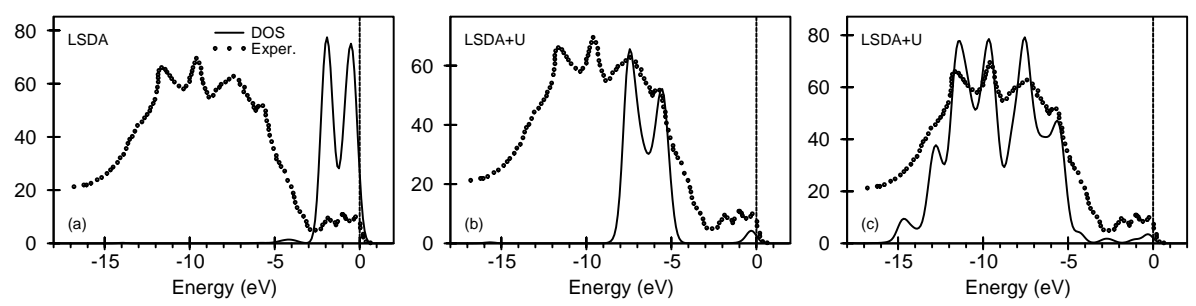

Figure 2. Comparison of the calculated $4 f$ DOS in LSDA and LSDA $+U$ approximations [8] with the experimental UPS spectra from [43]. The (c) panel shows the LSDA $+U$ if DOS taking into account the multiplet structure of the $4 f^{11}$ final state (see explanations in the text).

compound: it happens irrespective of the precise value of $U_{\text {eff }}$.

Photoemission experiments, both x-ray (XPS) and ultraviolet (UPS), have been of central importance for the understanding of mixed-valence materials (see the review of the early work by Campagna et al. [39]). In rare-earth photoemission, when the photon ejects an electron from the $4 f^{n}$ shell it leaves behind a $4 f^{n-1}$ configuration, hence the kinetic energy distribution curve of the emitted electron measures the spectra of the final-state hole. The final state $4 f^{n-1}$ has a characteristic multiplet splitting which serves as a fingerprint, and these are accurately resolved and calculable in rare-earth photoemission. By identification of the final-state hole the initial state can be inferred.

The partial $4 f$ DOS of the occupied part of the TmS calculated in LSDA and LSDA $+U$ approximations is compared with UPS measurements [41] in figure 2. The calculated $4 f$ DOS has been broadened to account for lifetime effects and for the experimental resolution. The Tm $4 p$ states do not essentially contribute to XPS and UPS due to the low ionization cross section compared with that of the Tm $4 f$ states [54]. Hence, the measurements only indicate the $f$ excitation energies of trivalent and divalent Tm, relative to the Fermi level. The LSDA calculations places the $4 f$ energy band right at the Fermi level (figure 1) producing in the $4 f$ DOS a double peak between 0 and $-3 \mathrm{eV}$ (figure 2). In the LSDA $+U$ calculations, twelve fully occupied $4 f$ bands are situated between -4 and $-8 \mathrm{eV}$ in the DOS and a partly filled $13^{\text {th }} 4 f$ band produces a small peak at around $-0.5 \mathrm{eV}$ binding energy (figure 2).

The LSDA $+U$ DOS cannot, of course, fully account for the multiplet splitting. Therefore, we present at the right panel in figure 2 the LSDA $+U 4 f$ DOS taking into account the multiplet structure of the $4 f^{11}$ final state. The multiplet structure consists of 15 terms nine of which are the most intensive $\left({ }^{4} \mathrm{I}_{15 / 2},{ }^{4} \mathrm{I}_{13 / 2},{ }^{4} \mathrm{~F},{ }^{2} \mathrm{H},{ }^{4} \mathrm{G},{ }^{2} \mathrm{~K}\right.$, ${ }^{4} \mathrm{D},{ }^{2} \mathrm{~L}$, and ${ }^{2} \mathrm{~F}$ ) [39]. The relative intensities for the multiplet peaks were obtained based on the Cox calculations [56] who used the fractional parentage method [57]. In this method the Hund's rule ground state is assumed for $n 4 f$ electrons and then the coefficients of fractional parentage (Racah's) for the $n-1$ configurations are calculated. The intensities for various configurations (multiplets) are just the square of the coefficients of fractional parentage. In the right panel in figure 2 UPS spectrum 

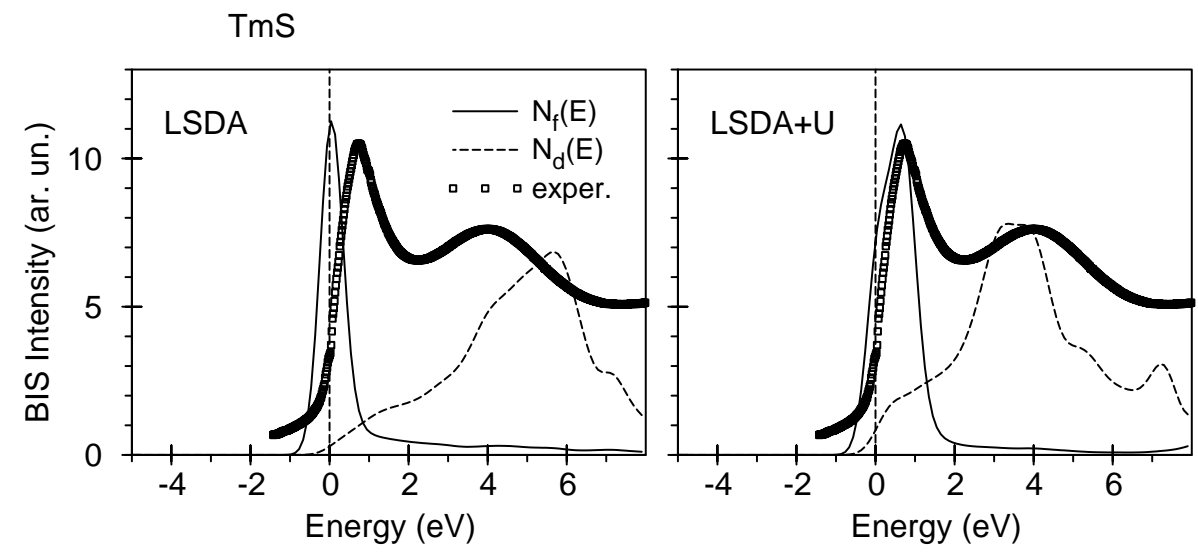

Figure 3. Comparison of the calculated partial $4 f\left(\mathrm{~N}_{f}(\mathrm{E})\right)$ and $5 d\left(\mathrm{~N}_{d}(\mathrm{E})\right)$ DOS in the LSDA and LSDA $+U$ approximations [8] with the experimental BIS spectrum of TmS from [55].

is modelled by a weighted sum of nine LSDA $+U 4 f$ DOS curves. The centroid of the DOS has been aligned with each term of the atomic final state multiplet, and summed up the spectra scaling them according to the relative intensities of the multiplets. The agreement between theory and the UPS measurements is very good. It is clear that the structures between -4.5 and $-16 \mathrm{eV}$ binding energy should be assigned to the final-state multiplet structure derived from twelve fully occupied $4 f$ bands $\left(\mathrm{Tm}^{3+}\right)$ and the structures between 0.0 and $-4.5 \mathrm{eV}$ are associated with the final-state multiplet structure of the partly occupied $13^{\text {th }} f$ level.

From the good agreement between theory and UPS measurements we may conclude that the LSDA $+U$ calculations give an accurate position for the occupied $4 f$ bands. The principal question is the energy position of the empty $4 f$ states, which is usually answered by x-ray bremsstrahlung isochromat spectroscopy (BIS) measurements. Figure 3 shows the experimental BIS spectrum of TmS [55] compared with the calculated energy distribution for the unoccupied partial Tm $4 f\left(\mathrm{~N}_{f}(\mathrm{E})\right)$ and $5 d$ $\left(\mathrm{N}_{d}(\mathrm{E})\right)$ density of states in the LSDA and LSDA $+U$ approximations. The experimental spectrum consists of two maxima at about $0.9 \mathrm{eV}$ and $4 \mathrm{eV}$ above the Fermi level. The first narrow peak was identified as having $f$ character, whereas the second broad peak was associated with $5 d-6 s$ states [55]. The LSDA places the empty $4 f$ states right at the Fermi level which contradicts the experimental data (figure 3). The LSDA $+U$ calculations place the maximum of the $14^{\text {th }} f$ hole level well above the Fermi level around $0.7 \mathrm{eV}$. The $13^{\text {th }} f$ hole level is partly occupied with its maximum DOS situated $0.025 \mathrm{eV}$ above the Fermi level (figure 1). Due to a rather large experimental resolution of the BIS spectra $(\approx 1 \mathrm{eV})$ [55] one experimentally observes essentially only a single BIS $4 f$ peak instead of two. However, a shoulder can be discerned split off from the main peak [55]. The LSDA $+U$ calculations give the correct position both for the $4 f$ and $5 d$ states (figure 3 ) within the experimental resolution. 

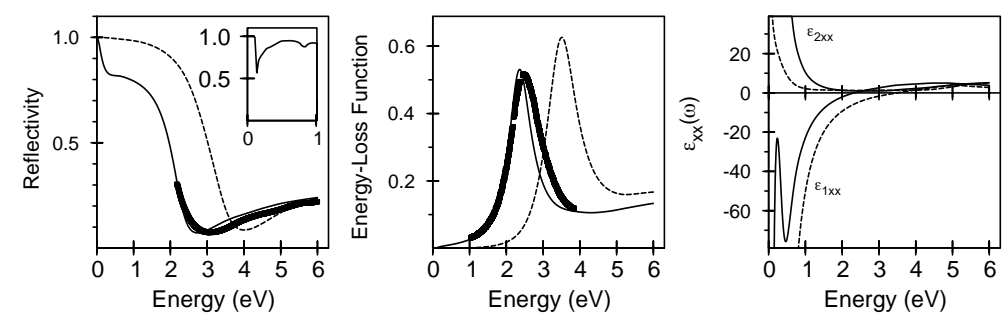

Figure 4. Calculated real and imaginary part of the diagonal dielectric function, $\varepsilon_{1 x x}, \varepsilon_{2 x x}$, energy-loss function and the optical reflectivity $R$ of $\mathrm{TmS}$ [8] treating $4 f$ states in core (dashed line) and by the LSDA $+U$ approximation (solid line) compared with experimental data (solid circles) [58].

Optical spectra More precise information on the band positions may be obtained from optical measurements. Although such measurements have much better resolution (the experimental resolution in optics is always in the meV range) in comparison with XPS and BIS, they produce complex functions containing information of both the initial and final states simultaneously (joint density of states) and are strongly effected by the optical transition matrix elements.

Figure 4 shows the calculated real and imaginary parts of the dielectric function, $\varepsilon_{1 x x}(\omega)$ and $\varepsilon_{2 x x}(\omega)$, the optical reflectivity and imaginary part of the energy-loss function $\operatorname{Im}[-1 / \varepsilon(\omega)]$ compared with experimental data [58]. Based on the results of the LSDA $+U$ band structure calculation, the observed optical reflectivity spectrum (figure 4) can be sorted into the respective interband transition groups: (1) metallic high reflectivity below $\approx 1 \mathrm{eV},(2)$ a steep edge between $\approx 1$ and $\approx 2.5 \mathrm{eV}$, and (3) above the minimum at $\approx 2.7 \mathrm{eV}$ some less pronounced structures with a broad maximum of $R$ between 5 and $7 \mathrm{eV}$ mostly caused by $3 p \rightarrow 5 d$ interband transitions.

The predominant structure of the TmS as well as TmSe reflectivity spectra is the edge at $2 \mathrm{eV}$. This sudden drop is characteristic of metallic rare-earth chalcogenides and is due to a plasma oscillation interfering with interband excitations [58]. This plasma resonance causes the golden color of TmS crystals as it does for all metallic rare-earth sulfides. The energy of the conduction electron plasma resonance in the presence of the interband excitations is given by $\varepsilon_{1 x x}(\omega)=0$. In the particular case of $\mathrm{TmS}$ this condition is fulfilled at $\hbar \omega=2.15 \mathrm{eV}$ and the maximum peak of the energy-loss spectrum in figure 4 is shifted only very little from this energy (by $-0.05 \mathrm{eV}$ ) as a result of damping. The correct energy position of the plasma edge in $\mathrm{TmS}$ can be obtained only by taking into account $5 d \rightarrow 4 f$ interband transitions. The calculations treating $4 f$ electrons as core electrons place the zero crossing energy of $\varepsilon_{1 x x}(\omega)$ at higher energies in comparison with the LSDA $+U$ calculations and as a result give a wrong energy position for the plasma resonance (figure 4). The insert of the figure 4 shows the unbroadened theoretically calculated optical reflectivity in the 0 to $1 \mathrm{eV}$ energy interval with two well pronounced minima at 0.11 and $0.9 \mathrm{eV}$. These features reflect the interband transitions from occupied $5 d$ states to empty $13^{\text {th }} f$ and $14^{\text {th }} f$ hole levels situated at 0.025 and $0.7 \mathrm{eV}$ respectively (figure 1 ). In real optical experiments such transitions are obscured by broadening from intraband 
Drude-like transitions, life-time effects and to a lesser extent by the experimental distortion.

\subsection{2. $\mathrm{TmSe}$}

The simplest argument for the TmS being trivalent, TmSe being intermediate valent and TmTe being divalent under normal conditions comes from comparing the lattice constants of rare-earth sulfides, selenides and tellurides as done by Bucher et al. [31]. The lanthanide contraction is the cause for the general trend in the curves and the standard divalent Sm, Eu and Yb ions with their larger ionic radius are the obvious deviations. TmTe lies on the divalent curve, TmS lies on the trivalent curve and TmSe is intermediate and thus by linear interpolation between a hypothetical divalent and trivalent TmSe one obtains a valence of $\mathrm{Tm}^{2.75+}$. Further evidence that TmSe is intermediate valence comes from x-ray and ultraviolet photoemission spectroscopy measurements [39,43]. The photoemission study of TmSe [43] gives the mean valence value equal to 2.79 and 2.68 from the study of the total yield spectra and off-resonance photoemission, respectively, although the mean valence of the same sample obtained from magnetic susceptibility measurement is 2.53 . We should mentioned that the total yield spectra reflect a more bulk information than photoemission spectra and the values obtained from transport properties or magnetic susceptibility are mostly bulk and not surface sensitive [43].

The ground state of an intermediate valence compound is a quantum mechanical mixture of both the $4 f^{n}$ and the $4 f^{n-1} 5 d$ configuration on each rare earth ion. Such compounds need theoretical consideration beyond the mean field one-particle $\mathrm{LSDA}+U$ approximation due to a possible configuration interaction between different $4 f$ valence states. Although such an interaction should be smaller in Tm compounds in comparison, for example, with mixed-valent Ce compounds due to the contraction of the $4 f$ wave function, it can lead to a spontaneous inter-configuration fluctuations (ICF), introduced first by Hirst [59]. As briefly discussed by Varma [60], at $T=0$, fluctuations could be either static or dynamic. In the static case the system is spatially "inhomogeneous" in the sense that at inequivalent sites different valence states are present. Examples among the rare earth compound are $\mathrm{Eu}_{3} \mathrm{~S}_{4}$ or $\mathrm{Eu}_{3} \mathrm{O}_{4}$ [39]. Such static charge "fluctuations" have been known for a long time in the $3 d-$ series; $\mathrm{Fe}_{3} \mathrm{O}_{4}$, magnetite, being a typical example (see [22] and references therein). In the dynamic case the system shows fast local fluctuations which give an intrinsic width to the $f$-levels. At any given site $4 f$ charge fluctuations between the two configurations occur on a time scale $\tau_{\mathrm{ICF}}$, the so-called inconfiguration fluctuation time. The system on time average is "homogeneous", i.e. all sites are equivalent. TmSe is known to be a homogeneous mixed-valence compound [39]. Experiments such as lattice constant and isomer shift measurements which probe the sample on a time scale much longer than $\tau_{\text {ICF }}$ will see only one intermediate configuration, but the experiments such as XPS or UPS measurements which take place in a time much shorter than $\tau_{\text {ICF }}$ (up to $10^{6}-10^{7}$ times shorter [40]) will see the instantaneous picture of a mixture of the ions in the two valence states. 

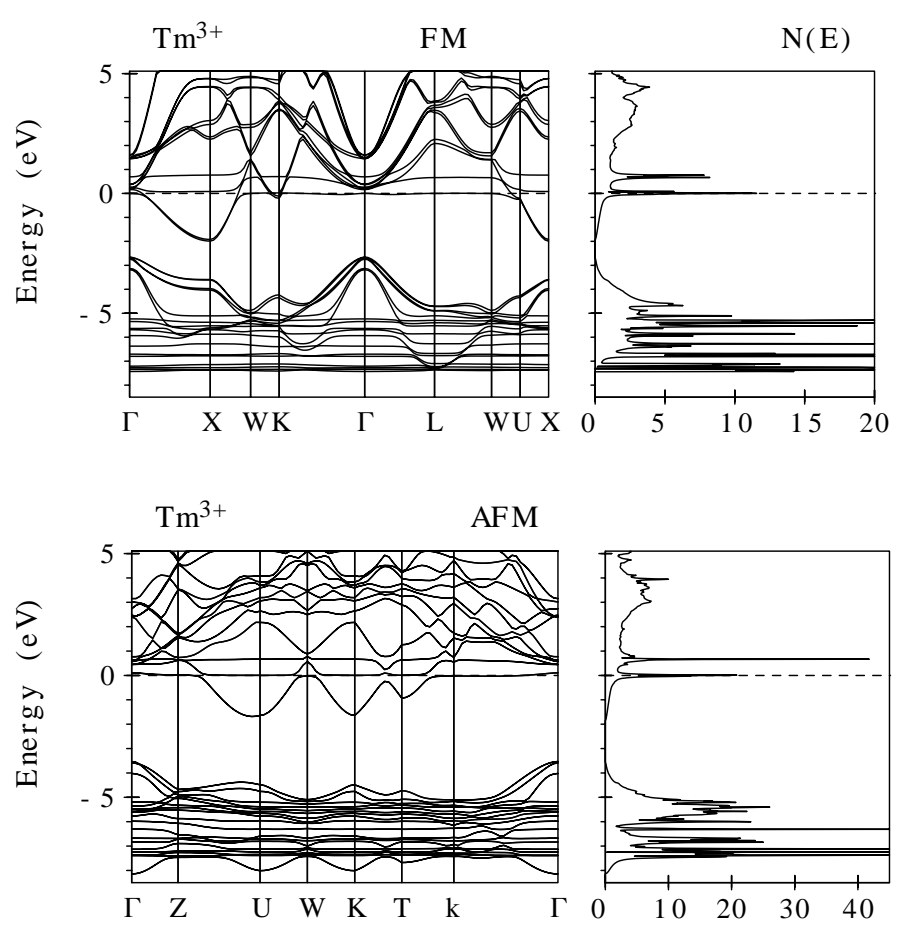

Figure 5. Self-consistent fully relativistic, spin-polarized energy band structure and total DOS (in states/(unit cell eV)) calculated for TmSe in ferromagnetic $(\mathrm{FM})$ and anti-ferromagnetic (AFM) ordering [8].

Band structure The LSDA $+U$ energy bands and total density of states of TmSe for $U_{\text {eff }}=6 \mathrm{eV}$ are shown in figure 5 both for the ferromagnetic and anti-ferromagnetic ordering for the $\mathrm{Tm}^{3+}$ ions. The electronic structure of $\mathrm{TmSe}$ for the $\mathrm{Tm}^{3+}$ ions is very similar to the TmS one with twelve $4 f$ bands fully occupied and hybridized with chalcogenide $p$ states. The $14^{\text {th }} f$ hole level is completely unoccupied and well above the Fermi level. A hole $13^{\text {th }} f$ level is partly occupied and pinned at the Fermi level. The occupation number of the $13^{\text {th }} f$ level is equal to 0.27 (valence $2.73+$ ) in good agreement with estimations from the lattice constants $(2.75+)$ as done by Bucher et al. [31] and from UPS measurements $(2.79+$ and $2.68+$ from total yield and photoemission spectra, respectively) [43].

Figure 6 shows the expanded view of the total DOS of TmSe and TmS both in the ferromagnetic (FM) and anti-ferromagnetic (AFM) states [8]. An important feature of the FM electronic structure is a high DOS at the Fermi energy $E_{\mathrm{F}}$ in both TmS and TmSe. A large DOS at $E_{\mathrm{F}}$ signals an instability with respect to metamagnetic phase transitions. Indeed, total energy calculations show that the ground state for TmSe as well as for $\mathrm{TmS}$ is an anti-ferromagnetic ordering in agreement with experiment $[34,47]$. Through the FM - AFM phase transition a pseudo gap at the Fermi level is opened in both TmS and TmSe (figure 6). The opening of such a pseudo gap may explain the increase of the electrical resistivity below Néel temperature [47,49,50], as well as an enormous negative magnetoresistivity associated with the metamagnetic transition to the high-field aligned state and the decrease of the Hall coefficient in 

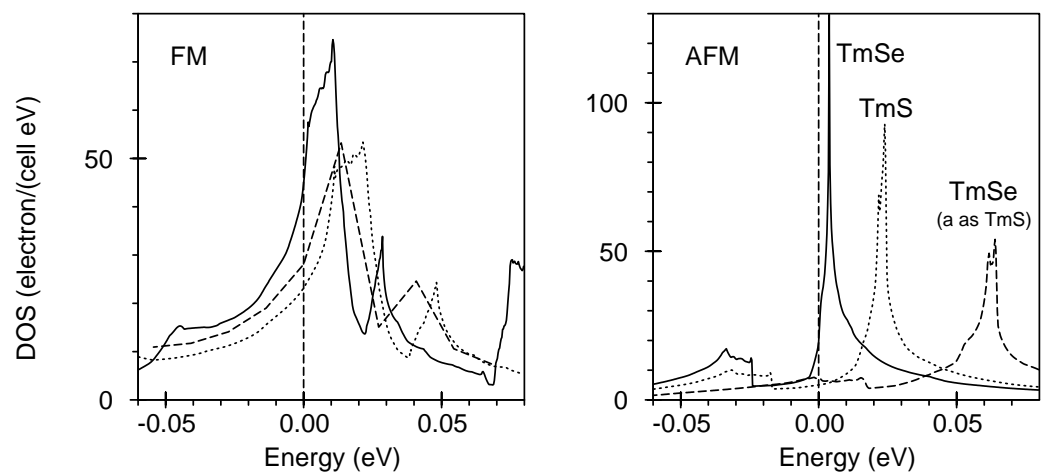

Figure 6. Expanded view of the DOS of TmSe (solid line), TmS (dotted line), and TmSe with the lattice constant of TmS (dashed line) calculated in the LSDA $+U$ approximation for ferromagnetic (FM) and anti-ferromagnetic (AFM) ordering $[8]$.

an external magnetic field [51].

The pinning of a partly occupied $13^{\text {th }} f$ level is different in TmS and TmSe. In contrast to TmS, the Fermi level crosses the $13^{\text {th }} f$ hole level in the vicinity of its DOS maximum in TmSe, both in the FM and the AFM states. The maximum DOS of the $13^{\text {th }} f$ hole level is situated above the Fermi level, as close as $4 \mathrm{meV}$ $(\approx 45 \mathrm{~K})$ in the AFM ground state of TmSe. The energy position of the $13^{\text {th }} f$ level is in a good agreement with low temperature far infrared optical measurements by Marabelli and Wachter [61]. According to their measurements the first interband transition appears at about $3 \mathrm{meV}$. We should mention here that when we speak about partial occupation of the $13^{\text {th }} f$ hole level in TmS we mean that such an occupation is due to the hybridization effect between $5 d$ and $4 f$ energy states. The peak position of the $13^{\text {th }} f$ hole level DOS in TmS is relatively far away from the Fermi level. A different situation occurs in TmSe where the Fermi level crosses the shoulder of the $13^{\text {th }} f$ hole level DOS (see figure 6 ), hence the $13^{\text {th }} f$ level is really pinned at the Fermi level. It is a typical situation for mixed-valent crystals.

The Tm monohalcogenides offer the unique opportunity to follow the evolution of intermediate valence as a function of composition. The driving force is the change of lattice constant upon exchanging the anion. In order to separate the effect of the lattice constant from the effect of the ionic potential of the chalcogenide component on the electronic structure of $\operatorname{TmX}(\mathrm{X}=\mathrm{S}$ and $\mathrm{Se})$, we also present in figure 6 the DOS of TmSe evaluated with the lattice constant of TmS [8]. The partial occupation of the $13^{\text {th }}$ hole $f$ level in this case is similar to the situation for TmS, although the energy positions of the maxima is not the same as in actual TmS. The mean valency of TmSe with the lattice constant of TmS is equal to $2.90+$, very close to the valency in actual $\mathrm{TmS}(2.88+)$. It can be considered as qualitative theoretical support of the conclusion derived in [53], that the application of pressure enhances the $\mathrm{Tm}^{3+}$ state relative to $\mathrm{Tm}^{2+}$ state in the TmSe.

In figure 7 the partial $4 f$ DOS of the occupied part of the TmSe band struc- 

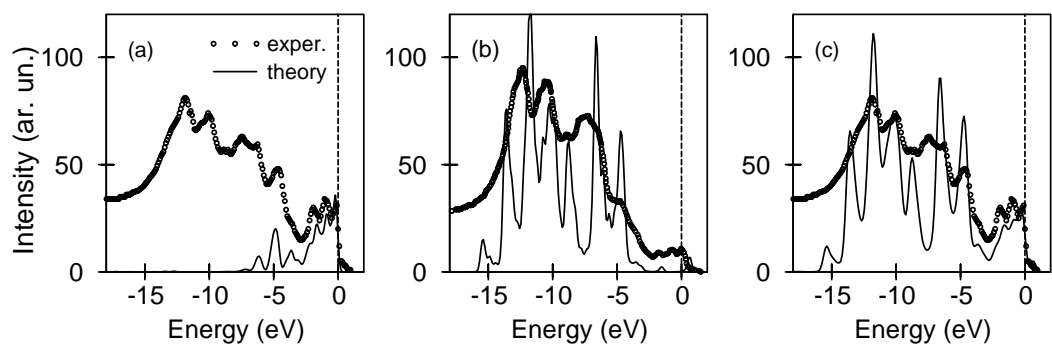

Figure 7. The calculated $\mathrm{Tm}^{3+}(\mathrm{a}), \mathrm{Tm}^{2+}$ (b) and $\mathrm{Tm}^{3+}+\mathrm{Tm}^{2+} 4 f$ (c) DOS of TmSe in LSDA $+U$ approximations [8], taking into account the multiplet structure of the $4 f^{12}$ and $4 f^{11}$ final states. Comparison is made with the experimental XPS spectra from [39] measured for $a=5.640 \AA(\mathrm{a})$ and $a=5.689 \AA(\mathrm{b}, \mathrm{c})$ lattice constants.

ture is compared with XPS measurements [39] for two single crystals with different lattice constants. An off-stoichiometric violet TmSe single crystal, with lattice constant $a=5.640 \AA$, contains almost entirely $\mathrm{Tm}^{3+}$ ions [39]. The theory calculations using a starting configuration with zero occupation of $14^{\text {th }}$ and $13^{\text {th }} f$ levels $\left(\mathrm{Tm}^{3+}\right.$ configuration) well describe this experimental XPS spectrum.

The XPS spectrum of TmSe with $a=5.689 \AA$, has a sizable divalent signal between 0 and $-3 \mathrm{eV}$ binding energy. The middle panel of figure 7 shows the theoretically calculated $4 f$ DOS of the $\mathrm{Tm}^{2+}$ Se taking into account the multiplet structure of the $4 f^{12}$ final state. The right panel shows the experimental XPS spectrum for $a=5.689 \AA$, in comparison with the sum of $\mathrm{Tm}^{3+}$ and $\mathrm{Tm}^{2+} 4 f$ DOSs in the proportion of fifty-fifty. These results suggest that the valency of the sample with $a=5.689 \AA$, is close to 2.5 as comes from the analysis of the DOSs. Of course it is difficult to make quantitative conclusions about the possible Tm $4 f$ configuration in TmSe from the comparison of theoretically calculated $4 f$ DOS with XPS or UPS spectra. From such a comparison we can only derive an energy position of finalstate multiplet structures from $\mathrm{Tm}^{3+}$ and $\mathrm{Tm}^{2+}$ ions. The XPS and UPS spectra are strongly affected by the transition matrix elements which have been omitted in the consideration for simplicity. Besides, the relative intensities of $\mathrm{Tm}^{3+}$ and $\mathrm{Tm}^{2+}$ ion final-state multiplet structures depend on the excitation photon energy. This was shown in [43] where the intensity ratio between the trivalent and divalent components in Tm monochalcogenides significantly changes depending on resonance or off-resonance conditions. Finally, UPS and XPS spectroscopies are strongly surface sensitive methods. The escape depth of a photoelectron with kinetic energy of a kilovolt is about $15 \AA$. For the softer radiation typical of the UPS measurements $(\leqslant 100 \mathrm{eV})$ the escape depth is only about $4 \AA[38]$, hence only the surface layers are probed. A recent advance in our understanding of mixed-valent photoemission comes from the recognition that surface spectra can be markedly different from that of the bulk. In particular, the valence at the surface can be very different [38].

In the optical reflectivity measurements the depth of penetration is given by the inverse absorption coefficient and typically amounts to $10^{2}$ to $10^{3} \AA$. This means 

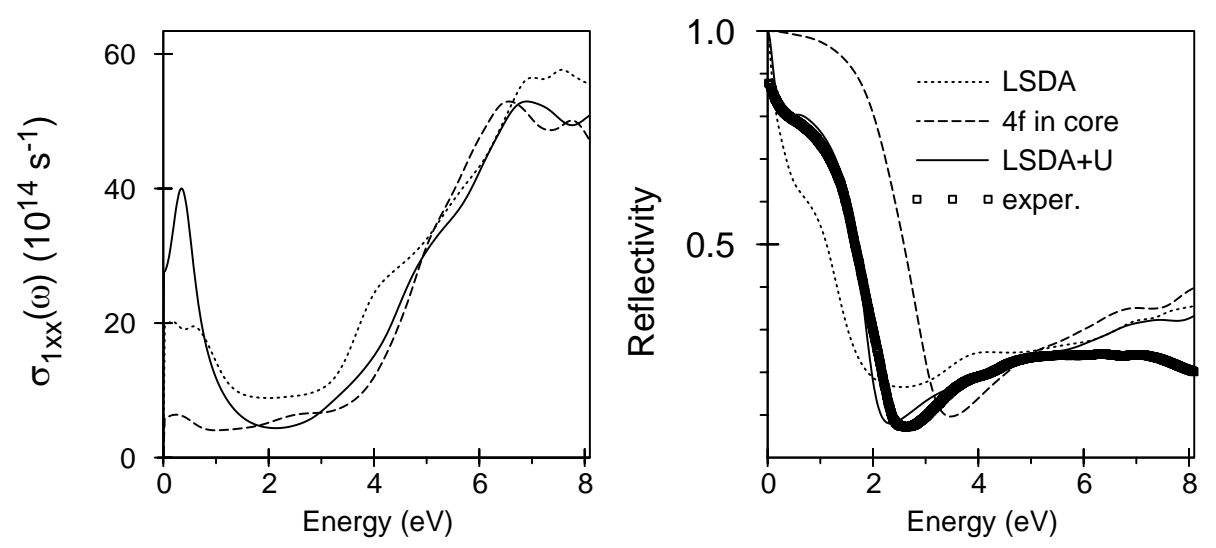

Figure 8. Calculated diagonal part of the optical conductivity $\sigma_{1 x x}$ and the optical reflectivity $R$ of TmSe [8] treating the $4 f$ states as: (1) fully localized ( $4 f$ in core) (dashed line); (2) itinerant (LSDA) (dotted line); and (3) partly localized $(\mathrm{LSDA}+U)$ (solid line) compared with the experimental data [58] (open squares).

that the optical measurements are not so surface sensitive.

Optical spectra Figure 8 shows the calculated diagonal part of the optical conductivity $\sigma_{1 x x}$ and the optical reflectivity of TmSe treating the $4 f$ states as: (1) fully localized ( $4 f$ in core); (2) itinerant (LSDA); and (3) partly localized (LSDA $+U$ ) in comparison with experimental data [58]. The calculations treating $4 f$ electrons as core electrons place the zero crossing energy of $\varepsilon_{1 x x}(\omega)$ at higher energies in comparison with the LSDA $+U$ calculations and as a result give a wrong energy position for the plasma resonance which determines a deep minimum in the optical reflectivity (figure 8). Due to the wrong position of $4 f$ energy bands in the LSDA calculations this approximation is incapable of producing the correct optical reflectivity in the infrared spectral region. Figure 8 clearly demonstrates that the better description of the optical reflectivity spectrum is given by the LSDA $+U$ approach. The $5 d \rightarrow 4 f$ interband transitions play an essential role in the fast decrease of the optical reflectivity in 0 to 0.5 eV energy interval.

\subsection{3. $\mathrm{TmTe}$}

Figure 9 shows the energy band structure and total density of states of TmTe calculated in LSDA and LSDA $+U$ approximations. Although TmTe is known as a magnetic semiconductor, LSDA calculations gave a metallic solution.

In contrast to LSDA, the LSDA $+U$ calculations predict a correct ground state of TmTe, namely, a magnetic semiconductor with direct energy gap of $0.86 \mathrm{eV}$ at the $\Gamma$ symmetry point and an indirect gap of $0.58 \mathrm{eV}$ between the top of $\mathrm{Tm} 4 f$ valence band at the $\Gamma$ point and the bottom of Tm $5 d$ conduction band at the $\mathrm{X}$ symmetry point. Theory produces a somewhat larger energy gap in comparison with the optical measurements of $0.35 \mathrm{eV}$ [32]. Thirteen completely occupied Tm $4 f$ energy bands 

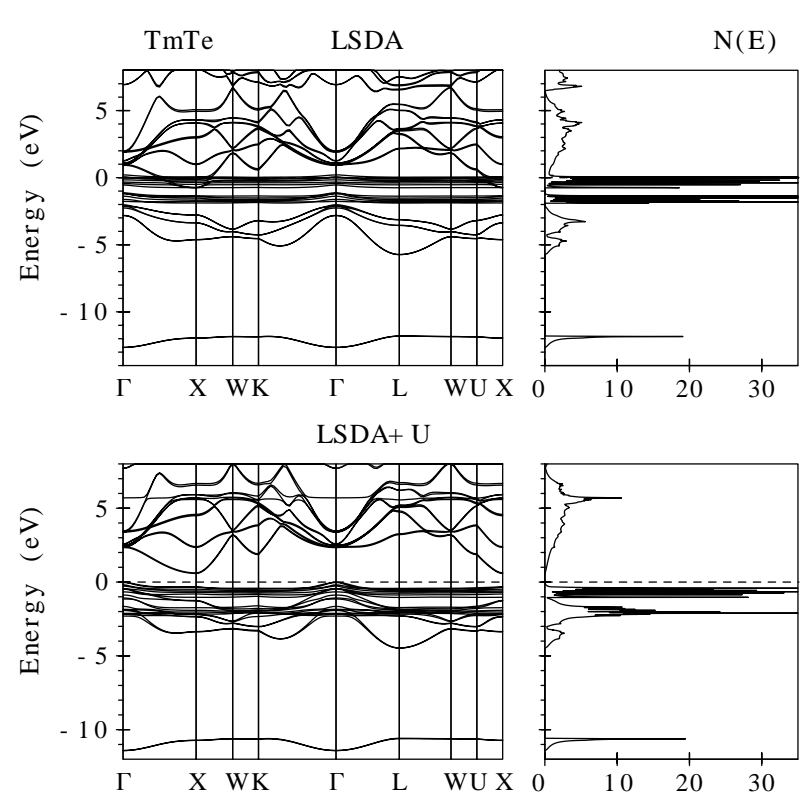

Figure 9. Self-consistent fully relativistic, spin-polarized energy band structure and total DOS (in states/(unit cell eV)) calculated for TmTe [8].

located between 0.0 and $-2.2 \mathrm{eV}$ are hybridized with Te $5 p$ states. The empty $14^{\text {th }}$ $f$ energy level is situated around $\approx 5.8 \mathrm{eV}$ above the Fermi level hybridized with Tm $5 d$ states (figure 9). In other words, theory predicts the Tm atom in TmTe to be in divalent state.

\subsection{Sm monochalcogenides}

The Sm monochalcogenides offer an interesting possibility to study the transition from semiconductor to the mixed valence (MV) state as a function of pressure. First, it is of great importance to characterize the semiconducting state.

The energy band structure of SmS presented in figure 10 with the $4 f$ electrons in the core can be subdivided into three regions separated by energy gaps. The bands in the lowest region around $-11 \mathrm{eV}$ have mostly $\mathrm{S} s$ character with some amount of $\mathrm{Sm} s p$ character mixed in. The next six energy bands are $\mathrm{S} p$ bands separated from the $s$ bands by an energy gap of about $7 \mathrm{eV}$. The width of the $\mathrm{S} p$ band is about $3.2 \mathrm{eV}$. The unoccupied electronic states can be characterized as Sm $5 d$ bands. The sharp peaks in the DOS calculated in the LSDA just below and above the Fermi energy are due to $4 f_{5 / 2}$ and $4 f_{7 / 2}$ states respectively (figure 10).

The LSDA $+U$ band structure calculations [16] have been started from a $4 f^{6}$ configuration for the $\mathrm{Sm}^{2+}$ ion with six on-site $4 f$ levels shifted downward by $U_{\text {eff }} / 2$ and eight levels shifted upwards by this amount. The energies of occupied $4 f_{5 / 2}$ and unoccupied $4 f_{7 / 2}$ levels are separated by approximately $U_{\text {eff }}$. The LSDA $+U$ energy bands and total density of states of $\mathrm{SmS}$ for $U_{\text {eff }}=6 \mathrm{eV}$ are shown in figure 10. The Coulomb repulsion $U_{\text {eff }}$ strongly affects the electronic structure of SmS. For $\mathrm{Sm}^{2+}$ ions six $4 f_{5 / 2}$ bands are fully occupied and situated in the gap between $\mathrm{S} p$ and $\mathrm{Sm}$ 

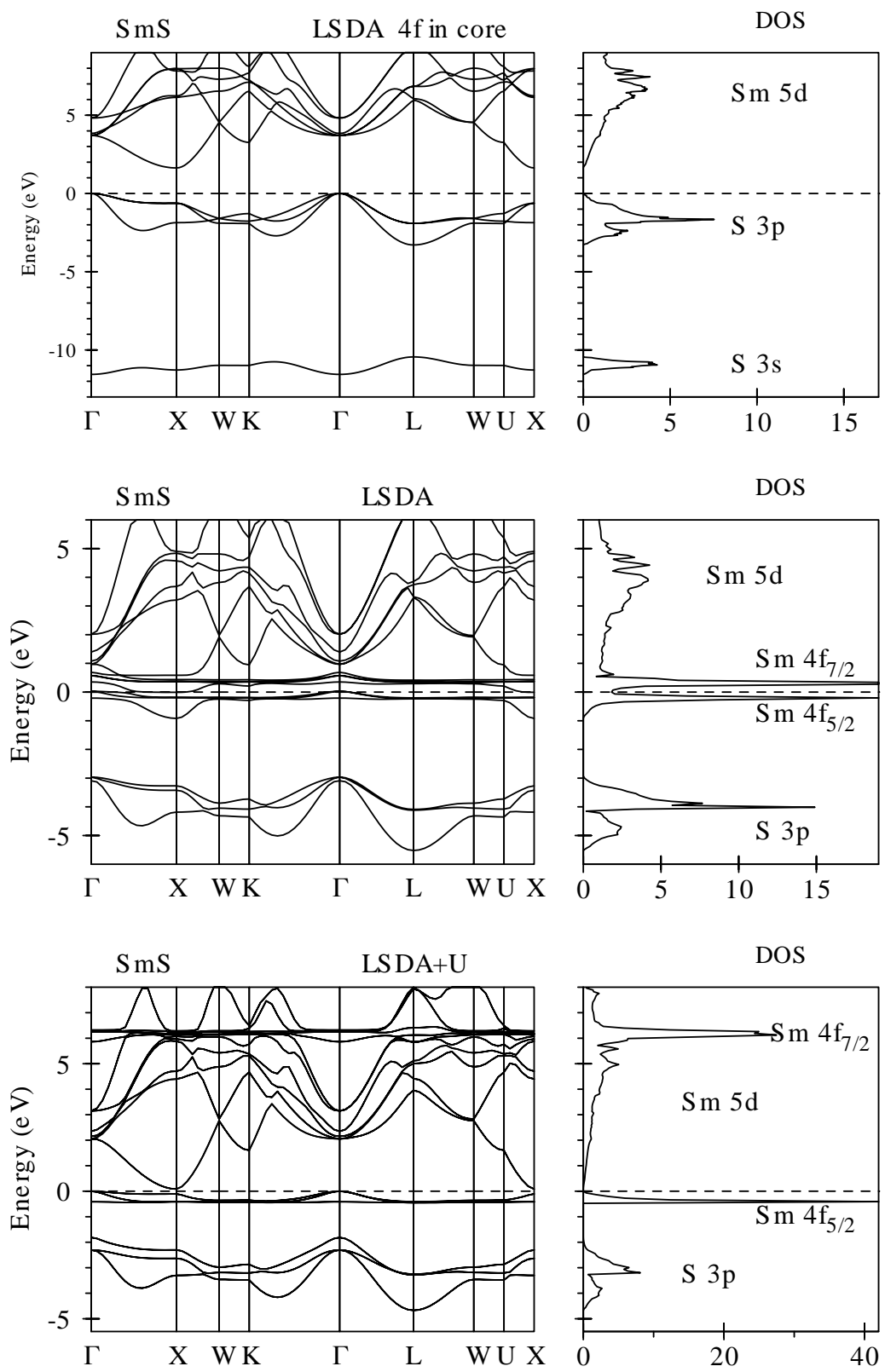

Figure 10. Self-consistent fully relativistic, spin-polarized energy band structure and total DOS (in states/(unit cell eV)) calculated for SmS [16] treating the $4 f$ states as: (1) fully localized ( $4 f$ in core); (2) itinerant (LSDA); and (3) partly localized $(\mathrm{LSDA}+U)$. 

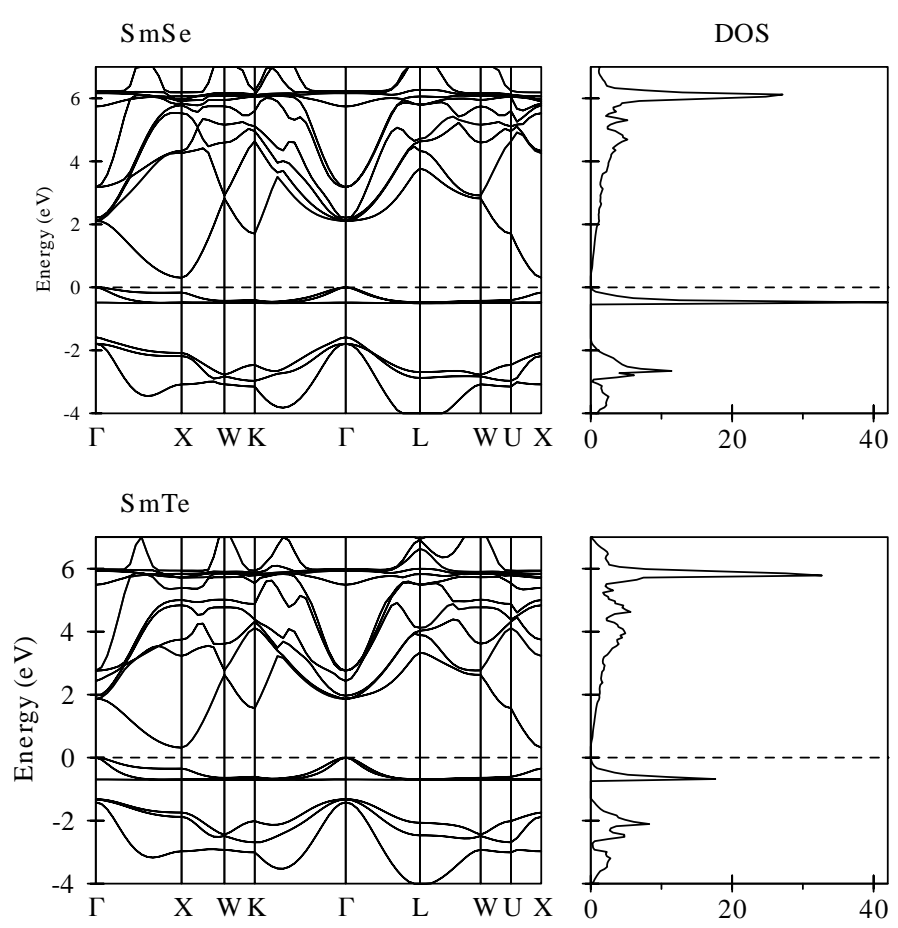

Figure 11. Self-consistent fully relativistic, spin-polarized energy band structure and total DOS (in states/(unit cell eV)) calculated for SmSe and SmTe in LSDA $+U$ approximation [16].

$5 d$ states while the $4 f_{7 / 2}$ hole levels are completely unoccupied and well above the Fermi level hybridized with $\mathrm{Sm} 5 d$ states which results in a non-magnetic ground state with the Sm ion in the divalent state. The theoretically calculated energy gap $\Delta E=0.18 \mathrm{eV}$ which is formed between $\mathrm{Sm} 4 f_{5 / 2}$ and $\mathrm{Sm} 5 d$ states is in good agreement with the experimentally estimated $0.15 \mathrm{eV}$ derived from the activation energy [33].

The LSDA $+U$ energy bands and total density of states of SmSe and SmTe for $U_{\text {eff }}=6 \mathrm{eV}$ are shown in figure 11 . Their electronic structures are very similar to the SmS one with six Sm $4 f_{5 / 2}$ bands fully occupied and the $4 f_{7 / 2}$ hole bands completely unoccupied and well above the Fermi level hybridized with Sm $5 d$ states. Theory gives energy gaps between $\mathrm{Sm} 4 f_{5 / 2}$ and $5 d$ bands equal to 0.47 , and $0.67 \mathrm{eV}$ in SmSe, and SmTe, respectively. The corresponding experimental values are equal to 0.45 and $0.65 \mathrm{eV}[33]$.

Photoemission experiments, both x-ray (XPS) and ultraviolet (UPS), have been of central importance to the understanding of mixed-valence materials. The partial $4 f$ DOS of the occupied part of the SmS calculated in LSDA and LSDA $+U$ approximations is compared with XPS measurements [62] in figure 12. The theoretically calculated $4 f$ DOS cannot, of course, fully account for the multiplet splitting. Therefore, figure 12 presents the $4 f$ DOS's taking into account the multiplet structure of the $4 f^{5}$ final state. This multiplet structure consists of 3 terms ${ }^{6} \mathrm{H},{ }^{6} \mathrm{~F}$, and ${ }^{6} \mathrm{P}[62]$. In figure 12 the XPS spectrum is modeled by a weighted sum of three $4 f$ DOS 

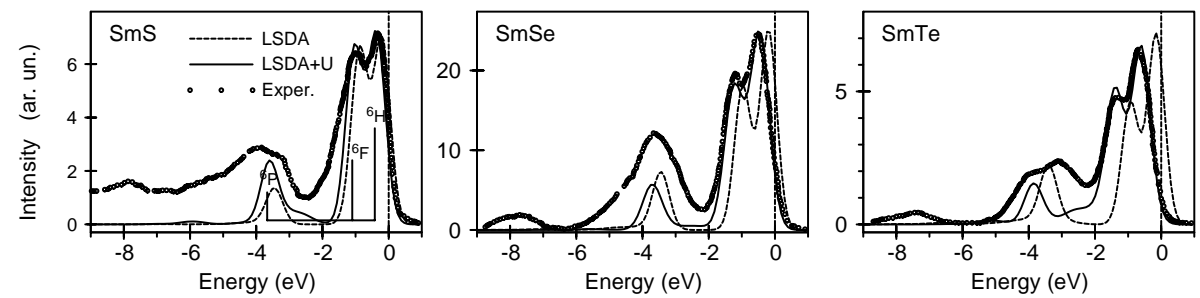

Figure 12. Comparison of the calculated $4 f$ DOS in LSDA and LSDA $+U$ approximations [16] with the experimental XPS spectra from [62] taking into account the multiplet structure of the $4 f^{5}$ final state (see explanations in the text).
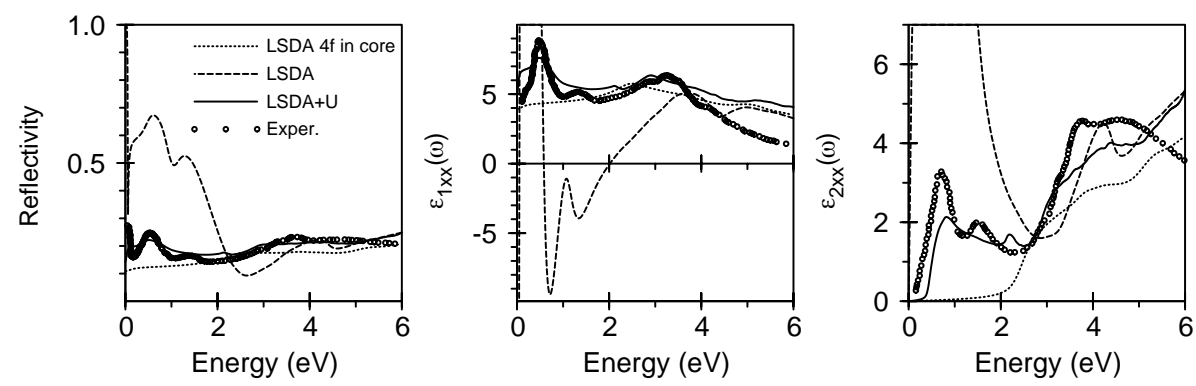

Figure 13. Calculated optical reflectivity $R$, real and imaginary parts of the diagonal dielectric function, $\varepsilon_{1 x x}, \varepsilon_{2 x x}$ of $\operatorname{SmS}[16]$ treating $4 f$ states as (1) fully localized ( $f f$ in core) (dotted line), (2) itinerant (dashed line) and (3) partly localized (LSDA $+U$ approximation) (solid line) compared with experimental data (open circles) [63].

curves. We aligned the centroid of the calculated occupied $4 f$ DOS peak with the centroid of the atomic final state multiplet. Although LSDA calculations produce almost the same picture as LSDA $+U$ calculations in the case of SmS, for SmSe and SmTe, the LSDA calculations place the $4 f_{5 / 2}$ energy bands too close to the Fermi level which leads to a disagreement with the measured XPS spectra (figure 12).

From the good agreement between theory and XPS measurements we may conclude that the LSDA $+U$ calculations give an accurate position for the occupied $4 f$ bands. The principal question is the energy position of the empty $4 f$ states, which is usually answered by optical or BIS measurements.

In figure 13 we show the experimental [63] real and imaginary parts of the dielectric function, $\varepsilon_{1 x x}(\omega)$ and $\varepsilon_{2 x x}(\omega)$, the optical reflectivity and optical conductivity $\sigma_{1 x x}(\omega)$ spectra, as well as the spectra calculated with LSDA, LSDA $+U$ and with the $4 f$ electrons in the core. This picture clearly demonstrates that a better description is unambiguously given by the LSDA $+U$ approach. As was mentioned above, the LSDA theory produces a metallic solution and, therefore, gives a wrong asymptotic behavior for the optical reflectivity and the dispersive part of the dielectric function $\varepsilon_{1 x x}$ as $\omega \rightarrow 0$. The most prominent discrepancy in the LSDA spectra is the extra peaks between 0 and $1.5 \mathrm{eV}$ in the $\varepsilon_{1 x x}(\omega), \varepsilon_{2 x x}(\omega)$ and optical conductivity 
$\sigma_{1 x x}(\omega)$ caused by interband transitions involving the occupied $4 f_{5 / 2}$ and unoccupied $4 f_{7 / 2}$ hybridized states. In the LSDA $+U$ approach, the empty $4 f_{7 / 2}$ state energies are shifted upward due to the on-site Coulomb interaction $U_{\text {eff }}$. As a result, the transitions involving the unoccupied $4 f_{7 / 2}$ states do not take place at small photon energies, and the erroneous peak structures around 0 to $1.5 \mathrm{eV}$ disappear from the optical spectra.

The calculations in which the $4 f$ electrons are treated as quasi-core are capable of reproducing correct asymptotic behavior for the optical reflectivity and the dispersive part of the dielectric function $\varepsilon_{1 x x}$ as $\omega \rightarrow 0$ similar to the LSDA $+U$ calculations, but, it fails in producing a peak at around $0.6 \mathrm{eV}$ in the absorptive part of the dielectric function $\varepsilon_{2 x x}$ and optical conductivity spectra. This peak is mostly determined by the $4 f \rightarrow 5 d$ interband transitions.

The LSDA $+U$ theory also gives good agreement between calculated and measured optical spectra in the cases of SmSe and SmTe [16] (not shown).

\subsubsection{High pressure golden phase of SmS}

The history of the Sm monochalcogenides as MV materials started at the beginning of the 1970's when Jayaraman et al. [64] and then Bucher et al. [65] discovered a pressure-induced semiconductor-metal transition and suggested that the metallic state would be mixed valent. It was a surprise that $\mathrm{SmS}$ showed this transition occurring at the incredibly low pressure of $6.5 \mathrm{kbar}$. Starting the pressure above the phase transition and decreasing it, a large hysteresis is observed and the MV state snaps back to a semiconductor state at $1.5 \mathrm{kbar}$. For SmSe and SmTe the pressureinduced valence transition is continuous and is completed at higher pressures, about 45 and 60 kbar, respectively [33].

By increasing external pressure and hence, decreasing the lattice constant, the widths of Sm $5 d$ and $4 f$ bands are increased. In addition, the crystal-field splitting of the $5 d$ states $e_{g}-t_{2 g}$ in $\mathrm{SmS}$ is also increased. At a given pressure the $5 d$ band overlaps with the $4 f_{5 / 2}$ states and the energy gap becomes zero (figure 14 ). It occurs at a lattice constant around $5.70 \AA$ [33]. Starting from the overlap of $4 f$ and $5 d$ states, $4 f$ electrons will spill into the $5 d$ band leaving a $4 f^{5}$ state behind. The ionic radius of $\mathrm{Sm}^{3+}$ is about $15 \%$ smaller than the radius of $\mathrm{Sm}^{2+}$, so that simultaneously with more electrons in the $5 d$ conduction band the lattice will shrink, thus further increasing the crystal-field splitting of the $5 d$ states, resulting in an avalanche effect and a first-order valence transition. However, the valence transition does not go all the way to trivalency, but stops where the gain in electronic energy is compensated by an increase of lattice strain energy [33].

The LSDA $+U$ energy bands and total density of states of $\mathrm{SmS}$ for $\mathrm{Sm}^{3+}$ are shown in figure 14 . There are five $4 f_{5 / 2}$ bands fully occupied and hybridized with the bottom of the $\mathrm{S} p$ states. The $4 f_{7 / 2}$ unoccupied states are well above the Fermi level. The initially empty $6^{\text {th }} 4 f_{5 / 2}$ hole level became partly occupied and pinned at the Fermi level in the process of self-consistent relaxation with the occupation number equal to 0.25 (valence $2.75+$ ). It is a typical situation for mixed-valent crystals. 

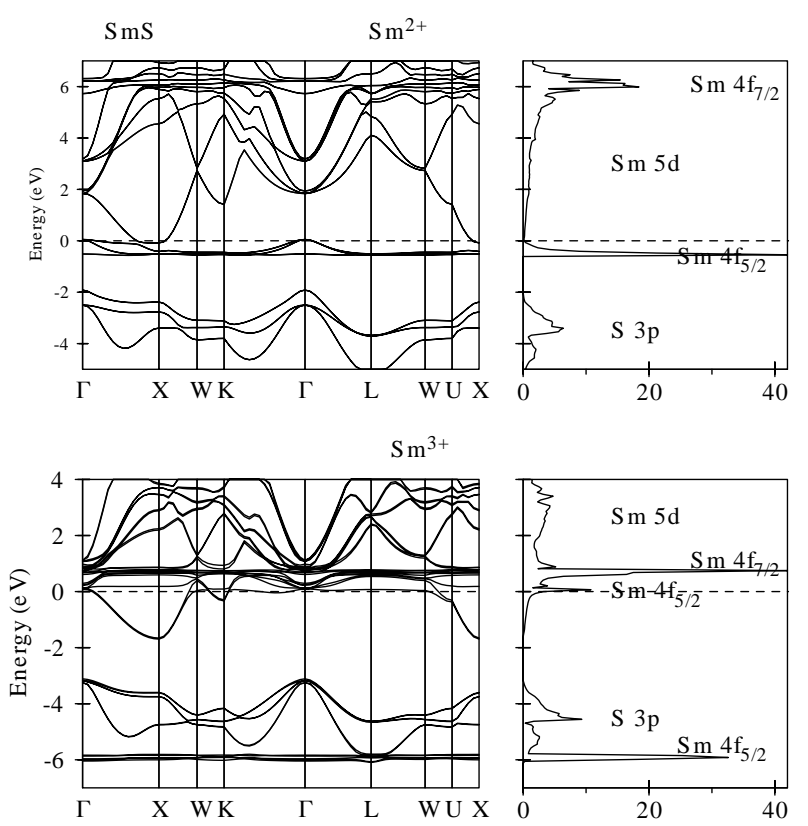

Figure 14. Self-consistent fully relativistic, spin-polarized energy band structure and total DOS (in states/(unit cell eV)) calculated for SmS with the LSDA $+U$ approximation for divalent and trivalent Sm atoms [16].

We can use two different representations in the construction of the LSDA $+U$ method, namely, $\left(j, m_{j}\right)$ and $\left(l, m_{l}\right)$ representations. Most of rare earths and their compounds have a rather large $4 f$ spin magnetic moment. Therefore, it is natural to use the $\left(l, m_{l}\right)$ representation in the calculations of their electronic structure. In this case one chooses the projection of the orbital momentum onto the spin direction $m_{l}$ for the occupied states. The SmS black phase as well as SmSe and SmTe have a nonmagnetic ground state. Therefore, it was used in that case the $\left(j, m_{j}\right)$ representation [16]. For fully occupied $4 f_{5 / 2}$ states the $z$ projections of the total moment were equal to $m_{j}=-5 / 2,-3 / 2,-1 / 2,1 / 2,3 / 2$, and $5 / 2$.

The situation is not clear for gold SmS. Both representations were used for the calculation of the electronic structure of the golden high pressure phase of SmS. Figure 14 presents the energy band structure of gold $\mathrm{SmS}$ in the $\left(j, m_{j}\right)$ representation with $m_{j}=-1 / 2$ for the hole state. Due to the existence of a hole in the $4 f_{5 / 2}$ shell the LSDA $+U$ gives a so-called low magnetic moment ground state with the total magnetic moment equal to $0.240 \mu_{\mathrm{B}}$ (spin and orbital moments have opposite directions with values equal to -0.305 and $0.545 \mu_{\mathrm{B}}$, respectively). The $\left(l, m_{l}\right)$ representation gives a high spin magnetic moment ground state in SmS with the total magnetic moment equal to $4.636 \mu_{\mathrm{B}}$ at each $\mathrm{Sm}^{3+}$ site (spin and orbital moments have opposite directions with values equal to 5.501 and $-0.865 \mu_{\mathrm{B}}$, respectively). We should mention, however, that although our LSDA $+U$ band structure calculations always give a nonzero samarium magnetic moment, in gold $\mathrm{SmS}$ all the efforts to find a magnetic superstructure in high pressure SmS using neutron experiments 
have remained unsuccessful. However, one cannot exclude either a weak magnetic component below the limit of the experimental sensitivity (samples for high pressure measurements are very tiny) or an incommensurate structure giving peaks at positions not searched in the neutron experiments. The evaluation of the magnetic ground state of gold SmS from first principles requires further investigation.

The pinning of a partly occupied $6^{\text {th }} 4 f$ level strongly depends on the lattice constant. The increase of the valency with decrease of the lattice constant which was found in our band structure calculations can be considered as qualitative theoretical support of the conclusion derived from various experimental measurements, [33] that the application of pressure enhances the $\mathrm{Sm}^{3+}$ state relative to $\mathrm{Sm}^{2+}$ state in $\mathrm{SmS}$. The theoretically calculated samarium valency was found to be equal to $2.55+$ and $2.86+$ for high and low spin solutions, respectively. The experimentally estimated one is about $2.6+$ using spectroscopic methods and susceptibility measurements [6668 ] and about 2.8+ using the Vegards-low analysis of lattice constant measurements [68].

Some experimental results indicate that the golden phase of $\mathrm{SmS}$ could be a narrow-gap semiconductor. Evidence for a gap comes from the activation behavior of the electrical resistivity and point contact measurements [33]. The estimations from the point-contact spectra shows a possible gap of about $6.4 \mathrm{meV}$ [33]. On the other hand, some experiments indicate that there may not be a gap but rather a pseudo gap, and the hybridization does not occur over the whole Brillouin zone. Although the temperature dependence of the resistivity in the gold phase of SmS is semiconductor-like, the resistivity is increased only one order of magnitude with cooling from room temperature to several K's, whereas e.g. in $\mathrm{SmB}_{6}$ it is 5 orders of magnitude [33]. Direct optical measurements of mechanically polished (high pressure gold phase) SmS by Travaglini and Wachter [69] shows that, in contrast to $\mathrm{SmB}_{6}$, the reflectivity does not tend to a constant value for $\omega \rightarrow 0$ but it seems to rise toward $100 \%$ as for a metal. Besides, there is a linear with $T \gamma$ term in the specific heat presumably due to conduction electrons [70]. The LSDA $+U$ band structure calculations of gold SmS [16] produce a pseudo gap at the Fermi level with a peak just above and a shoulder below the Fermi level with predominantly $f$ character. We should mention that when the density of states at $E_{\mathrm{F}}$ is small compared to the giant density of states of the $f$ peaks, the resistivity may nevertheless appear activated over a certain temperature range as experimentally observed, but metallic conductivity should persist for the lowest temperatures [69].

\section{3. $\mathrm{SmB}_{6}$}

$\mathrm{SmB}_{6}$ are classical mixed valence (MV) narrow gap semiconductors. This class of materials is characterized by their electronic properties, which at high temperatures are associated with a set of independent localized $(f)$ moments interacting with a sea of conduction electrons, while at low temperature the electronic properties resemble those of a narrow gap semiconductor. $\mathrm{SmB}_{6}$ is the first compound in which the phenomena of MV were detected directly by x-ray absorption [71]. Despite more than 35 years of experimental and theoretical effort, many fundamen- 
tal aspects of the microscopic description of the MV ground state and the nature of valence fluctuations are still under discussion [33,72,73]. The main problem is the origin of the MV ground state, which seems intimately connected with the gap formation. One question is whether Kondo insulating materials are true insulators at low temperatures or whether an intrinsic small conduction-electron carrier concentration is present [74]. Infrared absorption [75,76], inelastic neutron scattering [77-80], optical conductivity [81], electron tunnelling [82], and electrical transport [83-85] measurements all detect a small electronic gap $\Delta=3-20 \mathrm{meV}$. However, the low-temperature transport properties of $\mathrm{SmB}_{6}$ are manifestly metallic, having a large but finite resistivity below $\approx 4 \mathrm{~K}$ [83-85]. There is a considerable controversy whether these in-gap states are intrinsic and present in pristine $\mathrm{SmB}_{6}[77-80,86,87]$, or extrinsic and dictated entirely by sample quality [83-85,88,89]. The size of the energy gap determined by different methods, varies considerably. For example, the value of the gap in $\mathrm{SmB}_{6}$ is equal to $2.7 \mathrm{meV}$ by tunnelling spectroscopy [82], $5 \mathrm{meV}$ from the temperature dependence of the electrical resistivity [83-85], and $16 \mathrm{meV}$ by point-contact spectroscopy [90]. Direct measurements of the low-temperature dynamical conductivity and dielectric permittivity of single crystalline $\mathrm{SmB}_{6}$ give evidence for a $19 \mathrm{meV}$ energy gap and an additional narrow donor-type band lying only $3 \mathrm{meV}$ below the bottom of the upper conduction band [91].

The average valence of $\mathrm{Sm}$ in the hexaboride was established to be 2.56 at room temperature, and to vary slightly with temperature by measurements of x-ray absorption [71,92], lattice constant [93], x-ray photoemission [39] and Mössbauer isomer shift [94].

The energy band structures of $\mathrm{SmB}_{6}$ have been calculated by Yanase and Harima [95] in the local spin density approximation and in [17] using the LSDA $+U$ approximation.

The compound $\mathrm{SmB}_{6}$ has the $\mathrm{CaB}_{6}$ type crystal structure with space group Pn3m (No 221) and lattice constant equal to $4.1333 \AA$. The structure of samarium hexaboride may be viewed as a $\mathrm{CsCl}$-type lattice with the cesium replaced by samarium and the chlorine by a $\mathrm{B}_{6}$ octahedron. Figure 15 shows the energy band structure of $\mathrm{SmB}_{6}$ calculated within the LSDA and the LSDA $+U$ approximations [17]. The energy band structure of $\mathrm{SmB}_{6}$ with the LSDA approximation can be subdivided into three regions separated by energy gaps. The bands in the lowest region around -14 to $-16 \mathrm{eV}$ (not shown) are mostly of B $2 s$ character with some amount of Sm $s p$ character mixed in. The next group of energy bands are B $2 p$ bands separated from the $2 s$ bands by an energy gap of about $3 \mathrm{eV}$. The width of the $\mathrm{B} 2 p$ band is about $8.8 \mathrm{eV}$. The Sm $5 d$ bands are partly occupied. They are also separated from $\mathrm{B} 2 p$ states by the energy gap of around $0.7 \mathrm{eV}$. The sharp peaks in the DOS calculated in the LSDA just below and above the Fermi energy are due to $4 f_{5 / 2}$ and $4 f_{7 / 2}$ states respectively (figure 15). They cross the $\mathrm{Sm} 5 d$ bands and hybridize with them. There is a small direct energy gap at the Fermi level of around $23 \mathrm{meV}$.

The LSDA $+U$ energy bands and total DOS of $\mathrm{SmB}_{6}$ for $U_{\text {eff }}=7 \mathrm{eV}$ are shown in figure 15. The Coulomb repulsion $U_{\text {eff }}$ strongly effects the electronic structure of $\mathrm{SmB}_{6}$. For $\mathrm{Sm}^{3+}$ ions five $4 f_{5 / 2}$ bands are fully occupied and hybridized with the $\mathrm{B}$ 

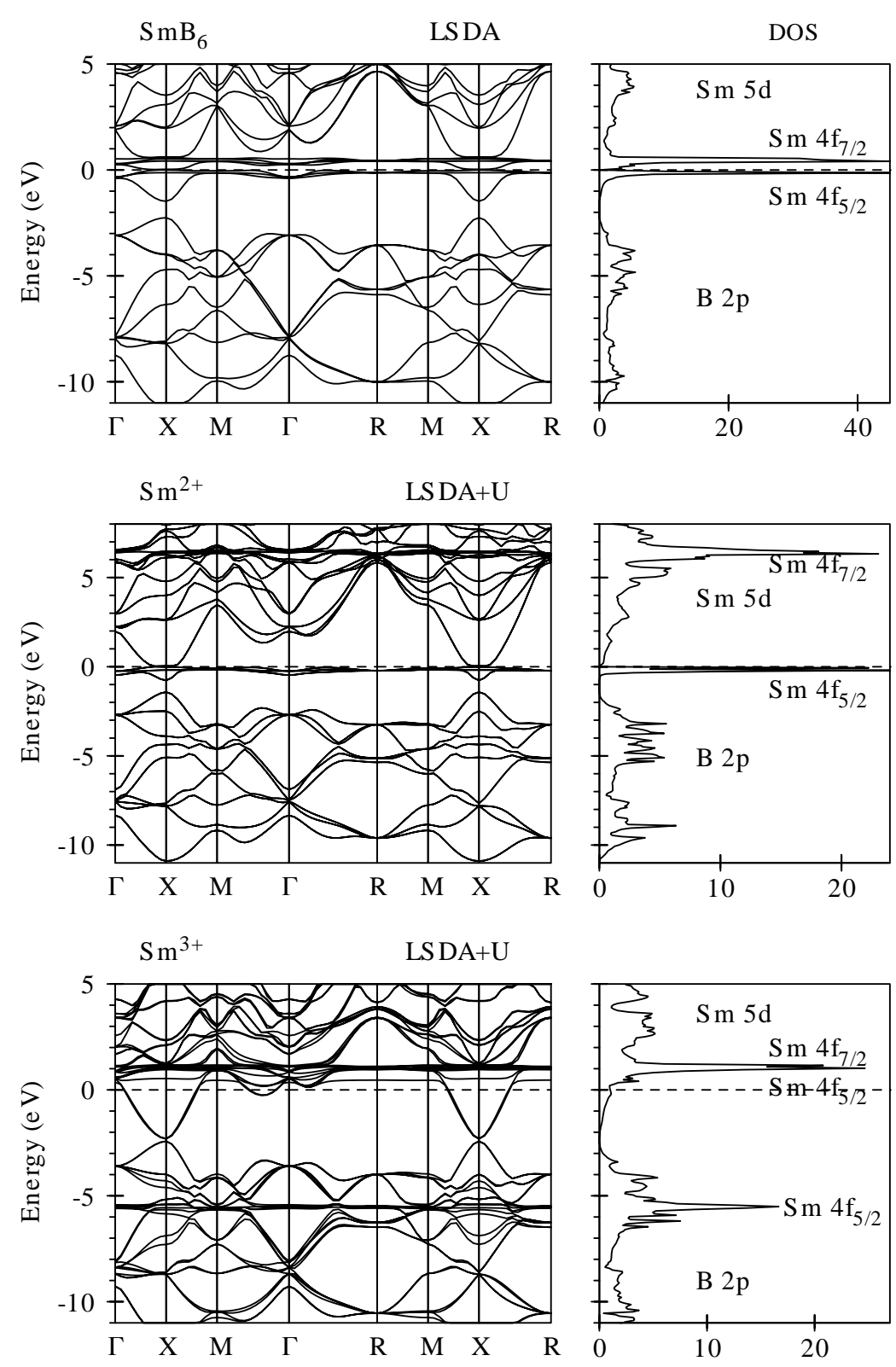

Figure 15. Self-consistent fully relativistic, spin-polarized energy band structure and total DOS (in states/(unit cell eV)) calculated for $\mathrm{SmB}_{6}$ with LSDA and LSDA $+U$ approximations [17].

$2 p$ states. The $4 f_{7 / 2}$ unoccupied states are above the Fermi level at around $1 \mathrm{eV}$. A single $4 f_{5 / 2}$ level is fully unoccupied and situated at about $0.34 \mathrm{eV}$ above the Fermi level.

The LSDA $+U$ band structure calculations [17] have been started from a $4 f^{6}$ configuration for the $\mathrm{Sm}^{2+}$ ion with six on-site $4 f$ energies shifted downward by $U_{\text {eff }} / 2$ and eight levels shifted upwards by this amount. The energies of occupied $4 f_{5 / 2}$ and unoccupied $4 f_{7 / 2}$ levels are separated by approximately $U_{\text {eff }}$ (figure 15 ). 

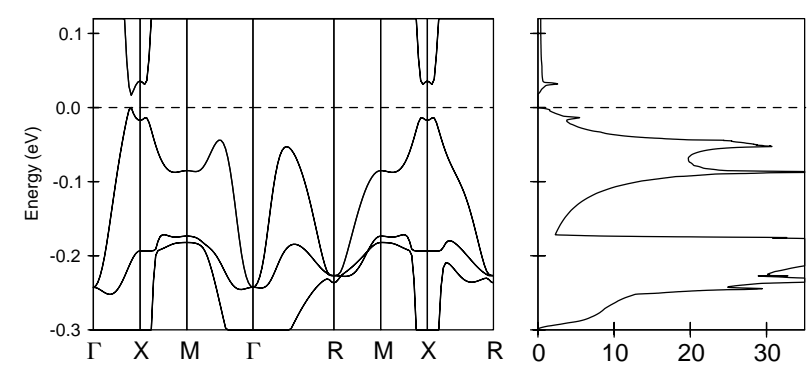

Figure 16. Expanded view of the energy band structure and total DOS (in states/(unit cell eV)) of $\mathrm{SmB}_{6}$ for $\mathrm{Sm}^{2+}$ ions calculated in $\mathrm{LSDA}+U$ approximations [17].
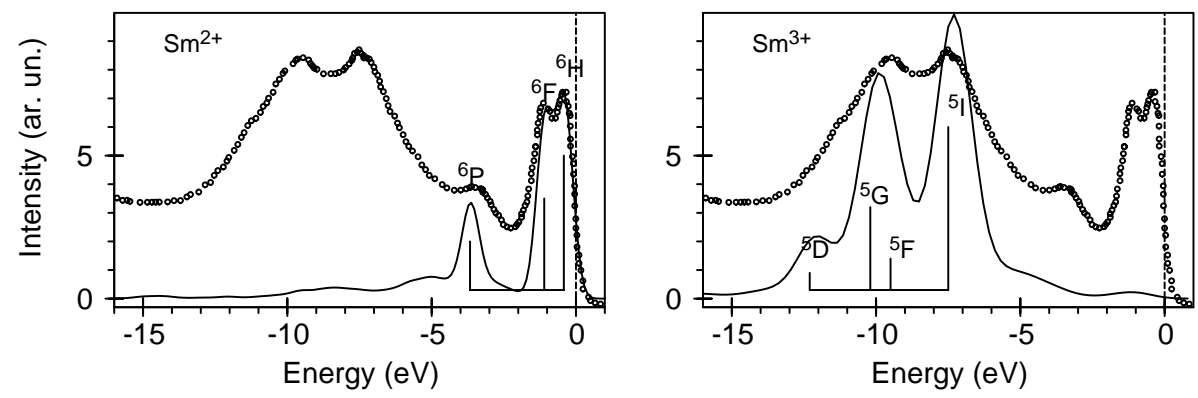

Figure 17. Comparison of the calculated $4 f \mathrm{DOS}_{\text {of }} \mathrm{SmB}_{6}$ using LSDA $+U$ approximation [17] with the experimental XPS spectra from [39] taking into account the multiplet structure of the $4 f^{5}$ and $4 f^{4}$ final states (see explanations in the text).

For $\mathrm{Sm}^{2+}$ ions eight $4 f_{7 / 2}$ hole levels are completely unoccupied and well above the Fermi level and hybridized with $\mathrm{Sm} 5 d$ states. The $4 f_{5 / 2}$ bands are situated in close vicinity of the Fermi level. They cross the bottom of Sm $5 d$ band. Figure 16 shows an expanded view of the LSDA $+U$ energy band structure and total DOS for $\mathrm{SmB}_{6}$ with $\mathrm{Sm}^{2+}$ ions. There is a hybridization gap at the Fermi level $\Delta E=27 \mathrm{meV}$. Although the LSDA $+U$ calculations [17] used a starting configuration with fully occupied $4 f_{5 / 2}$ bands, in the process of self-consistent relaxation, one of the $4 f_{5 / 2}$ doubly degenerate bands becomes partly unoccupied and is situated above the Fermi level around the $X$ symmetry point (figure 16) due to $\mathrm{Sm} 5 d-4 f$ hybridization. There is a small peak in the DOS above the Fermi level at around $0.03 \mathrm{eV}$ with predominantly $4 f$ character. This peak contains $0.024 f$ holes. In other words, the LSDA $+U$ calculations produce a semiconducting ground state in $\mathrm{SmB}_{6}$ with noninteger Sm valency equal to 2.02+.

The XPS spectra for $\mathrm{SmB}_{6}$ indicate a multiplet structure which can be identified with both $\mathrm{Sm}^{2+}$ and $\mathrm{Sm}^{3+}$ configurations being present. Figure 17 shows the $\mathrm{SmB}_{6}$ XPS spectrum [39] in comparison with the occupied part of the partial LSDA $+U$ $4 f$ DOS calculated with a multiplet structure of the final states taken into account in the same way as for $\mathrm{SmS}$ in previous section. We show in figure 17 the final state multiplet structure presented in [39]. $\mathrm{Sm}^{2+}$ ( $4 f^{5}$ final state) has the multiplets 


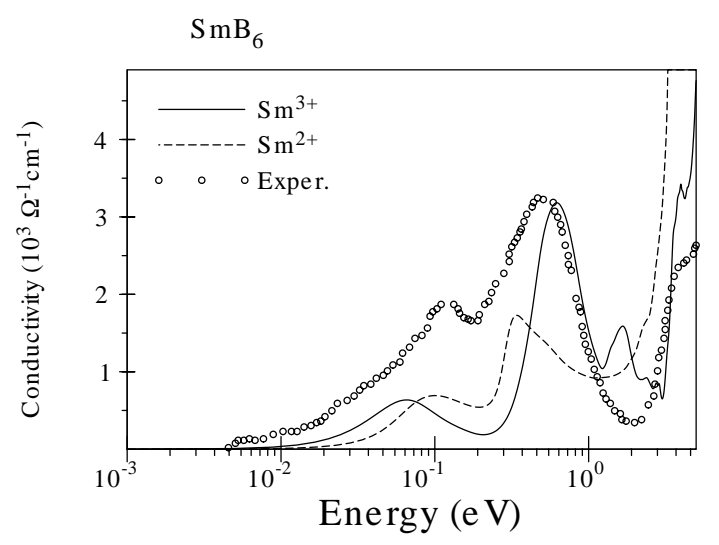

Figure 18. Calculated diagonal part of the optical conductivity $\sigma_{1 x x}(\omega)$ of $\mathrm{SmB}_{6}$ in the LSDA $+U$ approximation [17] compared with the experimental measurements at $13 \mathrm{~K}$ (open circles) [76].

${ }^{6} \mathrm{H},{ }^{6} \mathrm{~F}$, and ${ }^{6} \mathrm{P} . \mathrm{Sm}^{3+}\left(4 f^{4}\right.$ final state) has the multiplets ${ }^{5} \mathrm{I},{ }^{5} \mathrm{~F},{ }^{5} \mathrm{G}$, and ${ }^{5} \mathrm{D}[39]$. It is clear that the structures between 0.0 and $-5.5 \mathrm{eV}$ binding energy should be assigned to the final-state multiplet structure derived from six fully occupied $4 f$ bands $\left(\mathrm{Sm}^{2+}\right)$ and the structures between -5.5 and $-13 \mathrm{eV}$ are associated with the final state multiplet structure of the $\mathrm{Sm}^{3+}$ ions. The agreement with the positions of the multiplet peaks indicates that the LSDA $+U$ gives reasonably correct average positions for the occupied $4 f$ states.

Let us turn now to the optical properties of $\mathrm{SmB}_{6}$. Experimental investigation of the reflectivity spectra of $\mathrm{SmB}_{6}$ was performed by Kierzek-Pecold [96] in a narrow energy range from 1 to $5.6 \mathrm{eV}$. Thereafter, Travaglini and Wachter measured the reflectivity spectrum of $\mathrm{SmB}_{6}$ in the energy range from $1 \mathrm{meV}$ to $12 \mathrm{eV}$ at $300 \mathrm{~K}$ and $4 \mathrm{~K}$ to investigate the energy-gap [81]. Kimura with coworkers measured the optical spectra of all single crystal rare-earth hexaborides $R \mathrm{~B}_{6}(R=\mathrm{La}, \mathrm{Ce}, \mathrm{Pr}, \mathrm{Nd}$, $\mathrm{Sm}, \mathrm{Eu}, \mathrm{Gd}$, Th, Dy, Ho, Yb, and Y) in the energy region from $1 \mathrm{meV}$ to $40 \mathrm{eV}$ at $300 \mathrm{~K}$ and $13 \mathrm{~K}$ [76,97-99].

Figure 18 shows, with a logarithmic scale, the calculated optical conductivity

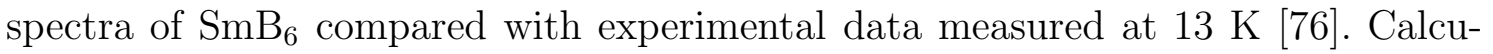
lations were performed for both the divalent and trivalent phases of $\mathrm{SmB}_{6}$. The experimentally measured optical conductivity spectrum has two strong peaks at 0.12 and $0.5 \mathrm{eV}$ and a steep rise at $3 \mathrm{eV}$ (figure 18). As might be expected, the experimental optical conductivity spectrum contains key features of both the divalent and trivalent theoretically calculated spectra. However, the three major peaks have different origins in the divalent and trivalent phases. A steep increase of optical conductivity starting at around $3 \mathrm{eV}$ is mostly determined by $\mathrm{B} 2 p \rightarrow \mathrm{Sm} 5 d$ interband transitions around the $\mathrm{X}$ symmetry point in the divalent samarium compound. The same feature in the trivalent phase can be described as a combination of the B $2 p \rightarrow \mathrm{Sm} 5 d$ and $\mathrm{Sm} 5 d \rightarrow \mathrm{Sm} 4 f$ interband transitions. These transitions also occur mostly around the $\mathrm{X}$ symmetry point (figure 15 ). The low energy peak at $0.12 \mathrm{eV}$ in 
the divalent phase can be explained as interband transitions between the occupied Sm $d-f$ mixed states and unoccupied Sm $5 d$ states near the $\mathrm{X}$ symmetry point. The same structure in the trivalent $\mathrm{Sm}$ phase is due to interband transitions among the hybridized Sm $5 d$ bands along $\Gamma-\mathrm{X}, \Gamma-\mathrm{M}, \mathrm{X}-\mathrm{M}$, and $\mathrm{X}-\mathrm{R}$ symmetry directions. This peak is shifted at around $0.1 \mathrm{eV}$ toward smaller energies in comparison with the experimental measurements. The peak at $0.5 \mathrm{eV}$ in the divalent phase arises from interband transitions between the occupied $\mathrm{Sm} d-f$ mixed states and unoccupied Sm $5 d$ states near the $\mathrm{X}$ symmetry point and $\Gamma-\mathrm{X}$ and $\mathrm{X}-\mathrm{M}$ symmetry directions. The same structure in the trivalent samarium phase is due to interband transitions between the occupied Sm $5 d$ states and the empty $6^{\text {th }} 4 f$ hole band along $\Gamma-\mathrm{X}$, $\Gamma-\mathrm{M}$, and $\mathrm{X}-\mathrm{M}$ symmetry directions.

As we mentioned above, the XPS measurements cannot distinguish between an inhomogeneously mixed or a homogeneously mixed compound, i.e. whether $\mathrm{Sm}^{2+}$ and $\mathrm{Sm}^{3+}$ ions are integer valent and statically mixed or the charge between them just fluctuates slower than the time resolution of XPS. The experimental optical conductivity spectrum can be also considered as a mixture of both the divalent and trivalent spectra. From other experiments, $\mathrm{SmB}_{6}$ is known to be a homogeneous mixed-valence semiconductor compound with a small energy gap [33]. Mössbauer isomer shift vs. temperature measurements for $\mathrm{SmB}_{6}$ by Cohen et al. clearly show that above $700 \mathrm{~K}$ and down to $4.2 \mathrm{~K}$ the degree of valence mixing is a constant of around 2.56 [94]. The LSDA $+U$ band structure calculations [17] produce a metallic state with trivalent samarium ions if we start the self-consistent procedure with $\mathrm{Sm}^{3+}$. On the other hand, if we used fully occupied $4 f_{5 / 2}$ levels $\left(\mathrm{Sm}^{2+}\right)$ as a starting configuration we are able to produce a correct semiconductor ground state for $\mathrm{SmB}_{6}$ with a small hybridization energy gap, and a theoretically calculated valency $2.02+$. A comprehensive theory for the MV state is needed to provide a quantitative measure of mixing between two configurational states. The LSDA $+U$ calculations are only capable of providing specific details for individual configurational states.

\section{4. $\mathrm{Yb}_{4} \mathrm{As}_{3}$}

The rare-earth pnictides $\mathrm{Yb}_{4} \mathrm{X}_{3}(\mathrm{X}=\mathrm{P}, \mathrm{As}, \mathrm{Sb}, \mathrm{Bi})$ exhibit unusual physical properties [100-107]. The high-temperature phases of these compounds have the cubic anti- $\mathrm{Th}_{3} \mathrm{P}_{4}$ structure with space group $\mathrm{I} 4 \overline{3} \mathrm{~d}$. The Yb-ions occupy the P-sites with Wyckoff positions $12 \mathrm{a}, \overline{4} \ldots, x=0.069$, and the X-ions occupy the Th-sites with special Wyckoff positions 16c, .3., fixed by symmetry [108]. All Yb-atoms are aligned with four families of chains oriented along four diagonals of the cubic unit cell [109]. All four ytterbium pnictides have anomalously large lattice constants $(a=8.56,8.788,9.322$, and 9.573 $\AA$ for $\mathrm{X}=\mathrm{P}, \mathrm{As}, \mathrm{Sb}$, and Bi, respectively) [100,101]. Since the pnictide ion is trivalent, three of the four $\mathrm{Yb}$ ions have a filled $4 f$ shell (valency $2+$ ) while one ion is in a $4 f^{13}$ (valency $3+$ ) configuration. When all $\mathrm{Yb}$ sites are equivalent, the hole in the $4 f$ shell moves between different $\mathrm{Yb}$ ions and the system is a mixed valent metal. Its average $\mathrm{Yb}$-valence $Z=2.25$ is rather close to the observed one $\left(Z_{\exp }=2.20\right)$, obtained from Mössbauer experiments [102].

The most interesting material is $\mathrm{Yb}_{4} \mathrm{As}_{3}$. At about $T_{s}=300 \mathrm{~K}$ it undergoes a 
weak first-order phase transition with a trigonal distortion. This transformation was clearly established by susceptibility measurements [100], Mössbauer spectra [102], polarized neutron diffraction [110], and elastic constant measurements [111]. At the structural transformation, the crystal shrinks in a volume-conserving way along one of the four Yb-chain directions $(\langle 111\rangle$, say). The resulting trigonal unit cell with the trigonal angle $\alpha=90.8^{\circ}$ has the short chain along its main diagonal. The centered trigonal space group is now R3c with two inequivalent $\mathrm{Yb}$ sites at Wyckoff positions $2 \mathrm{a}, 3$. , and two further inequivalent $\mathrm{Yb}$ sites at Wyckoff positions $6 \mathrm{~b}, 1$, as well as two inequivalent As sites at Wyckoff positions $6 \mathrm{~b}, 1$. The distortion is accompanied by charge ordering with $\mathrm{Yb}^{3+}$ ions occupying the short chains [107]. So, the $4 f$ electronic state of $\mathrm{Yb}_{4} \mathrm{As}_{3}$ changes from a valence fluctuating state in the cubic phase to a charge-ordered state $\mathrm{Yb}^{3+} \mathrm{Yb}_{3}{ }^{2+} \mathrm{As}_{3}^{3-}$ in the trigonal phase, although the charge ordering is not complete $[102,110]$. The $\mathrm{Yb}^{2+}$ ions have a closed $4 f$ shell with no spin magnetic moment. The $\mathrm{Yb}^{3+}$ ions have the $4 f^{13}$ configuration with spin magnetic moment equal to one. Inelastic neutron scattering experiments on $\mathrm{Yb}_{4} \mathrm{As}_{3}$ revealed the existence of low-energy excitations that are characteristic of a one-dimensional anti-ferromagnetic coupling at low temperatures [110].

Below $100 \mathrm{~K}, \mathrm{Yb}_{4} \mathrm{As}_{3}$ exhibits a heavy fermion behavior: the resistivity shows a $T^{2}$ behavior ascribed to electron-electron interaction involving the heavy quasiparticle band, the Sommerfeld coefficient $\gamma$ of the electronic specific heat $C_{\mathrm{el}}$ has a relatively high value of about $200 \mathrm{~mJ} \cdot \mathrm{mol}^{-1} \mathrm{~K}^{-2}$, and the extrapolated magnetic susceptibility at $0 \mathrm{~K}$ has a rather large value $\chi(0) \simeq 3 \times 10^{-2} \mathrm{emu} / \mathrm{mol}$ (see [100]). The Sommerfeld-Wilson ratio is found to be of the order of unity, indicating Fermiliquid behavior. Below $2 \mathrm{~K}$ and for moderate applied magnetic fields $(>2 \mathrm{~T})$, the "high field" magnetic susceptibility of $\mathrm{Yb}_{4} \mathrm{As}_{3}$ drops considerably as compared to the low field value [112]. There is also a rapid decrease observed in the specific heat at an applied field of about $1 \mathrm{~T}$ [103], both observations indicating the destruction of the heavy electron behavior.

The Hall coefficient $R_{\mathrm{H}}$ of $\mathrm{Yb}_{4} \mathrm{As}_{3}$, extrapolated to $T=0$, is equal to $7 \times$ $10^{18} \mathrm{~cm}^{-3}$ (see [100]). It would correspond to a carrier concentration of about 0.001 per formula unit if only one type of carriers were present.

The pressure effects on transport properties of $\mathrm{Yb}_{4} \mathrm{As}_{3}$ were investigated by Okunuki et al. [113] and by Mori et al. [114]. A clear increase of the carrier concentration is observed with an increasing pressure which leads to a rapid decrease of the resistivity $\rho$. The maximum in the temperature dependence of both, $\rho$ and $R_{\mathrm{H}}$ also decreases with pressure. However, the inverse Hall mobility, i.e., the resistivity divided by the Hall coefficient, is found to increase with the increasing temperature without any anomaly in the low temperature phase even under high pressure. This demonstrates that the mobility is not affected by pressure.

Summarizing all the experimental data, we can conclude that there is a strong dependence of the transport properties of $\mathrm{Yb}_{4} \mathrm{As}_{3}$ on doping, pressure and sample preparation but the specific heat and magnetic susceptibility do not change very much. This observation suggests that $\mathrm{Yb}_{4} \mathrm{As}_{3}$ is not an ordinary heavy fermion system. The latter is usually interpreted within the context of a Kondo lattice model. 
The heavy quasiparticle bands are thought to originate from Kondo resonance states at the Fermi level which evolve into coherent band states below a characteristic temperature. However, this mechanism leads to a similar behavior of the specific heat coefficient $\gamma$ and the carrier number as functions of doping. As explained above, the opposite behavior is observed which is a strong argument against the Kondo scenario in this class of compounds.

The electronic structure of $\mathrm{Yb}_{4} \mathrm{As}_{3}$ has been experimentally investigated by means of photoemission [115], x-ray bremsstrahlung isochromat spectroscopy [116], optical properties [117-119]. The optical measurements $[117,118]$ show a strong temperature dependence of the optical reflectivity in the far infra-red energy region below $10 \mathrm{meV}$. The plasma frequency $\omega_{\mathrm{p}}$ derived from optical measurements [119] is very small. It varies from $0.08 \mathrm{eV}$ for $10 \mathrm{~K}$ to $0.14 \mathrm{eV}$ for $70 \mathrm{~K}$ once more indicating an extremely small carrier number. Photo-electron spectroscopy in the x-ray and ultraviolet ranges (XPS and UPS) provide information on the energy position of $\mathrm{Yb}^{2+}$ and $\mathrm{Yb}^{3+} 4 f$ states in charge ordered $\mathrm{Yb}_{4} \mathrm{As}_{3}$. Structures in UPS and XPS spectra with binding energy between 0 and $4 \mathrm{eV}$ were identified [115] to be the spinorbit doublet of the $4 f^{13}$ final states and the structures between 6 and $13 \mathrm{eV}$ to be the multiplet structures of the $4 f^{12}$ final states. The low temperature photoemission also does not show any evidence for Kondo resonance states close to the Fermi level, confirming the exclusion of the Kondo scenario for $\mathrm{Yb}_{4} \mathrm{As}_{3}$.

Two approaches have been proposed to explain the properties of $\mathrm{Yb}_{4} \mathrm{As}_{3}$. One is based on a one-electron picture and was applied by Ochiai et al. [100] in the form of a four-band model and by Kasuya [120] in the form of a two-band model. A characteristic feature of this approach is a hole band of As $4 p$ states and an Yb $4 f$ band with a high density of states near the Fermi level. The second approach treats the $4 f$ electrons as being strongly correlated [121-123]. Charge ordering of the $\mathrm{Yb}^{3+}$ ions and the associated structural phase transition are a consequence of reducing the sum of the Coulomb repulsion and exchange to a minimum and are described by a band Jahn-Teller effect of correlated electrons. The observed heavy-fermion behavior is ascribed to spin-like excitations in the $\mathrm{Yb}^{3+}$ chains.

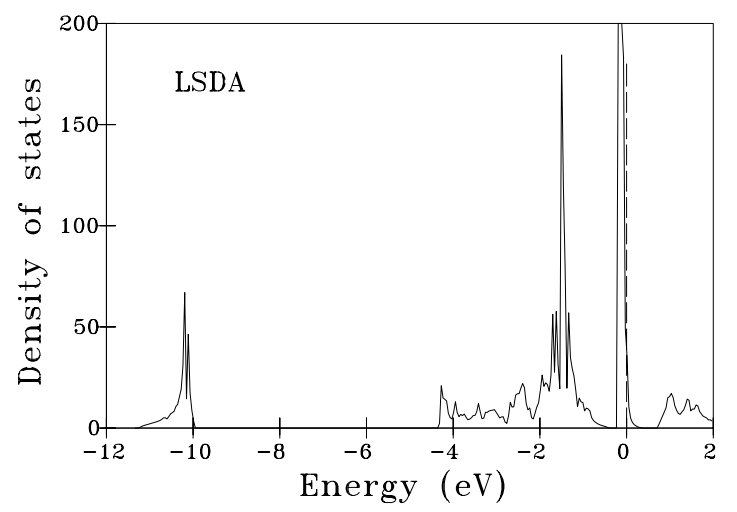

Figure 19. LSDA total DOS of $\mathrm{Yb}_{4} \mathrm{As}_{3}$ [14] (in states per cell and eV). 
These two approaches have been combined in [14] by studying the electronic structure and the physical properties of $\mathrm{Yb}_{4} \mathrm{As}_{3}$ in the trigonal low temperature $\mathrm{R} 3 \mathrm{c}$ phase within an $a b$ initio band-structure approach which takes strong correlations into account. This was achieved by applying a local spin density approximation to the density functional theory supplemented by a Hubbard $U$ term $(\mathrm{LSDA}+U)$. The electronic structure of the related compounds $\mathrm{Yb}_{4} \mathrm{X}_{3}\left(\mathrm{X}=\mathrm{P}, \mathrm{Sb}, \mathrm{Bi}\right.$, and $\left.\mathrm{P}_{0.5} \mathrm{As}_{0.5}\right)$ was also considered.

The LSDA total DOS of $\mathrm{Yb}_{4} \mathrm{As}_{3}$ is shown in figure 19. These results agree well with previous band structure calculations by Takegahara and Kaneta [124] and by Harima [95]. The occupied part of the valence band can be subdivided into three regions separated by energy gaps. The bands in the lowest region are mostly of As $s$ character with some amount of $\mathrm{Yb} s$ character mixed in. The next group of bands is formed by As $p$ states with little admixture of $\mathrm{Yb} p$ and $d$ states. The large narrow peak close to the Fermi energy is formed by $\mathrm{Yb} 4 f$ states. Unoccupied $5 d$ bands of $\mathrm{Yb}$ are separated from the $4 f$ states by an energy gap, which is a characteristic and important feature of the ytterbium pnictides. The position of the LSDA $4 f$ states close to the Fermi energy is, on the other hand, in contradiction to the findings of XPS and UPS experiments [115].

The LSDA $+U$ band structure calculations in [14] were started from the $4 f^{14}$ configuration for three $\mathrm{Yb}^{2+}$ ions, where all 14 on-site $4 f$ energies are shifted downwards by $U_{\text {eff }}^{(2+)} / 2$, and from the $4 f^{13}$ configuration for one $\mathrm{Yb}^{3+}$ ion with 13 on-site $4 f$ energies shifted downwards by $U_{\text {eff }}^{(3+)} / 2$ and one level shifted upwards by this amount. The energies of occupied and unoccupied $\mathrm{Yb}^{3+} f$ bands are separated by approximately $U_{\text {eff }}^{(3+)}$.

The LSDA $+U$ energy bands of $\mathrm{AF} \mathrm{Yb}_{4} \mathrm{As}_{3}$ for $U_{\mathrm{eff}}^{(2+)}=5.3 \mathrm{eV}$ and $U_{\mathrm{eff}}^{(3+)}=8.8 \mathrm{eV}$ are shown in figure 20. For three divalent $\mathrm{Yb}$ ions the $4 f$ bands are fully occupied and hybridize with As $p$ states in the energy range between -0.5 and $-2.5 \mathrm{eV}$. They are split due to spin-orbit coupling by $\Delta \epsilon_{\mathrm{so}}=1.40 \mathrm{eV}$. For the trivalent $\mathrm{Yb}$ ion, thirteen $4 f$ electron bands per ion are well below the Fermi level and separated
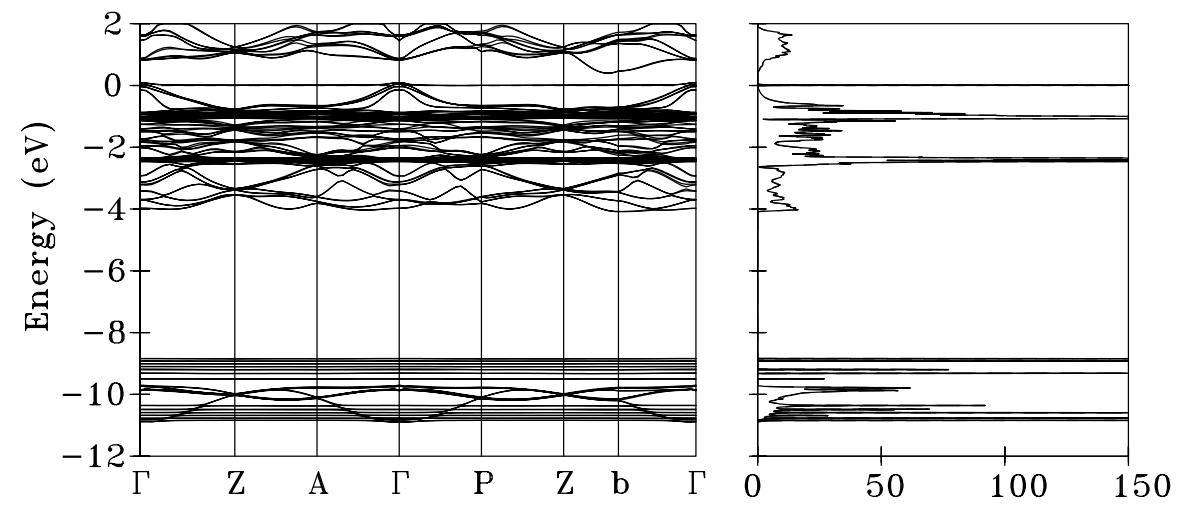

Figure 20. Self-consistent LSDA $+U$ energy band structure and total DOS (per unit cell and eV) of $\mathrm{Yb}_{4} \mathrm{As}_{3}[14] .\left(U_{\text {eff }}^{(2+)}=5.3 \mathrm{eV}, U_{\text {eff }}^{(3+)}=8.8 \mathrm{eV}\right)$ 


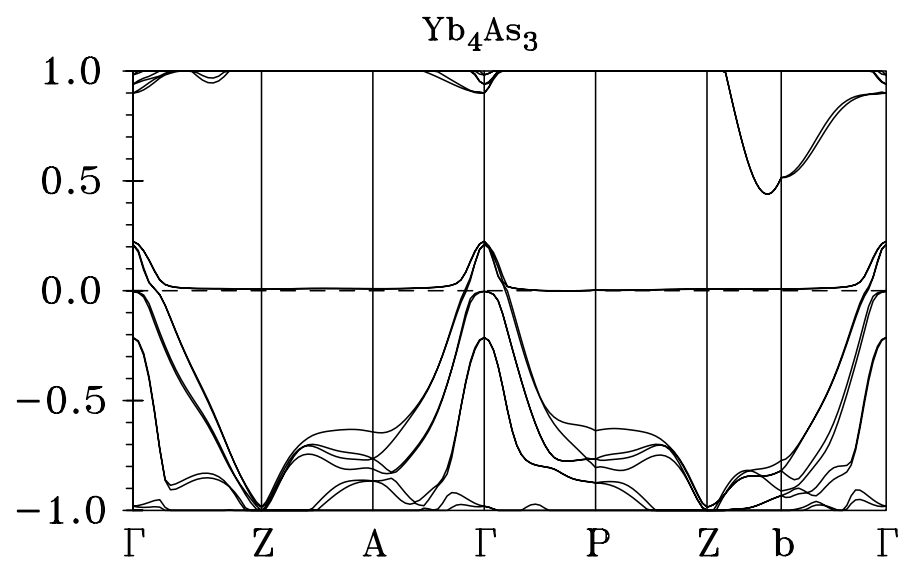

Figure 21. Self-consistent LSDA $+U$ energy band structure of $\mathrm{Yb}_{4} \mathrm{As}_{3}$ along the high-symmetry directions and for energies close to the Fermi level [14]. (Here, $U_{\text {eff }}^{(3+)}=9.6 \mathrm{eV}$, and the $f$ shell of the $\mathrm{Yb}^{2+}$ ions is treated as a core shell.)

from a $4 f$ hole state by the correlation energy $U_{\text {eff }}^{(3+)}$. One $4 f$ hole band per $\mathrm{Yb}^{3+}$ ion, doubly degenerated due to AF ordering, appears closely below the top of the As $p$ band. Since the As $p$ band is separated from the $Y b 5 d$ band by an ordinary band gap, the Fermi level should be pinned at the bottom of the $4 f$ hole band. This feature in combination with the small mass of the As $p$ electrons close to the top of the $p$ band appears to be a clue feature for the physics of the ytterbium pnictides. Due to the very small phase space for hybridization and the very small $\mathrm{Yb} 4 f-$ As $p$ orbital overlap, the DOS peak of the hole band is as narrow as $0.007 \mathrm{eV}$ right above the Fermi level. It is now clear why the usual Kondo lattice scenario is inappropriate for these pnictide compounds. For a Kondo resonance to develop, both the occupied and empty $4 f$-states should be sufficiently far away from the Fermi level. Quite opposite to this situation the (almost) empty level is pinned slightly above the Fermi energy. But due to the very small phase space for hybridization around the $\Gamma$-point, one still has an almost stable moment of the nearly integer occupation of the $f$-shell.

Since the $4 f$ binding energy of the $\mathrm{Yb}^{2+}$ ions is larger than $0.5 \mathrm{eV}$ and hence larger than the $\mathrm{Yb} 4 f-$ As $p$ orbital hybridization energy both in experiment and in the calculated band structure, the $4 f$ shell of the $\mathrm{Yb}^{2+}$ ions has been treated as a core shell in the following detailed numerical analysis. It has been checked that this simplification does not affect the issue. Therefore, we are left with a charge balance between $\mathrm{Yb} 4 f$ states of the $\mathrm{Yb}^{3+}$ ions and pnictide $p$ states only. Any charge transfer between these states causes a change in the Hartree potential which is governed by the bare Coulomb integral $U_{f}$ of the $4 f$ state being as large as $30 \mathrm{eV}$. Thus, an artificial shift via tuning $U_{\text {eff }}^{(3+)}$ of the hole $f$ level by $3 \mathrm{eV}$, say, (corresponding to a change of $U_{\text {eff }}^{(3+)}$ itself by $6 \mathrm{eV}$ ) will be completely compensated by a charge transfer as small as 0.1 electron charge. For this reason the $4 f$ hole level, to which the Fermi level is pinned, is itself very rigidly pinned close to the top of the pnictide $p$ band.

In figure 21 the band structure in the vicinity of the Fermi level is shown for 
$U_{\text {eff }}^{(3+)}=9.6 \mathrm{eV}$. It corresponds to a ground state with a hole pocket around the $\Gamma$-point $(k=0)$ holding 0.0058 As $p$ holes per formula unit. For the chosen values $U_{\mathrm{eff}}^{(3+)}=5.3,7.0,9.6$, and $9.8 \mathrm{eV}$, the obtained hole numbers are 0.0717, 0.0225, 0.0058 , and 0.0005 , respectively. Only for $U_{\text {eff }}^{(3+)} \geqslant 10 \mathrm{eV}$ the As $p$ band would be filled and the Fermi level would lie in a gap between this band and the $f$ hole state. This generic feature is obtained independent of whether the filled $f$ shell of the $\mathrm{Yb}^{2+}$ ions is treated as core or as valence, whether AF of ferromagnetic order is assumed on the $\mathrm{Yb}^{3+}$ chains, or whether spin-orbit coupling is taken into account or not, all the involved energies being small compared to the unscreened $U_{f}$.

The carrier density in $\mathrm{Yb}_{4} \mathrm{As}_{3}$ at a low temperature is extremely low, for which the following findings give evidence: (1) a large value of the resistivity up to $10 \mathrm{~m} \Omega$ [100], (2) a very low plasma frequency obtained from optical reflectivity [119], (3) a large value of the Hall coefficient [100] which corresponds to about 0.001 holes per $\mathrm{Yb}^{3+}$ ion, assuming the single carrier model. $\mathrm{Yb}_{4} \mathrm{As}_{3}$ is a compensated semimetal, so the number of As $p$ holes exactly equals the number of excess $\mathrm{Yb} 4 f$ electrons in the partially filled $4 f$ hole level. However, the mobility of heavy $4 f$ electrons is assumed to be negligible in comparison with the mobility of As $p$ electrons [100]. So, the transport properties of $\mathrm{Yb}_{4} \mathrm{As}_{3}$ are mostly determined by the number of As $p$ holes. In the analysis of [14], a carrier number of 0.001 per formula unit would result from a $U_{\text {eff }}^{(3+)}$ value between 9.6 and $9.8 \mathrm{eV}$ which would not be in contradiction to photoemission.

The small mass at the top of the pnictide $p$-band results in a very low $p$-DOS of 0.015 states per eV, atom and spin, at the $f$ hole level for $U_{\text {eff }}^{(3+)}=9.6 \mathrm{eV}$. That means that there are very few $p$-band states to broaden the $f$ level by hybridization. The width of the hole state equals to about $0.007 \mathrm{eV}(80 \mathrm{~K})$ and decreases with increasing $U_{\text {eff }}^{(3+)}$. The total DOS at the Fermi level, essentially resulting from the $4 f$ hole states, yields for $U_{\text {eff }}^{(3+)}=9.6 \mathrm{eV}$ a large band structure contribution to the Sommerfeld constant of $\gamma=\pi^{2} k_{\mathrm{B}}^{2} N_{\mathrm{A}} N\left(\epsilon_{\mathrm{F}}\right) / 3=40 \mathrm{~mJ} \cdot \mathrm{mol}^{-1} \mathrm{~K}^{-2}$. The calculated $\gamma$ is substantial, yet it corresponds only to the experimental value in a sufficiently strong magnetic field which suppresses magnetic moment fluctuations [103]. Since $\mathrm{Yb}_{4} \mathrm{P}_{3}$, although being a semiconductor, has an even larger value of the Sommerfeld constant than $\mathrm{Yb}_{4} \mathrm{As}_{3}$, a large part of $\gamma$ should be due to fluctuations of the magnetic moments in charge ordered $\langle 111\rangle$ chains. Beyond LSDA $+U$, further many-body interactions need to be invoked to account for the full Sommerfeld constant of the specific heat $[121,125]$.

\section{Summary}

We consider the electronic structure of some strongly correlated systems, such as $\mathrm{Tm}$ and $\mathrm{Sm}$ monochalcogenides, $\mathrm{SmB}_{6}$ and $\mathrm{Yb}_{4} \mathrm{As}_{3}$. It was shown that while the standard LSDA approach is incapable of correctly describing the electronic structure of these materials due to the strong on-site Coulomb repulsion, the LSDA $+U$ approach is remarkably accurate in providing a detailed agreement with experiment 
for a number of properties.

For the $\mathrm{Tm}^{3+}$ ions of TmS and TmSe, twelve $4 f$ bands are fully occupied and hybridize with chalcogenide $p$ states. The $14^{\text {th }} f$ hole level is completely unoccupied and well above the Fermi level. The initially empty hole $13^{\text {th }} f$ level in the process of self-consistent relaxation becomes partly occupied with $4 f$ DOS maximum situated in close vicinity of the Fermi level in TmS and TmSe. A fundamental aspect of this observation is that we find a partial occupation of the $13^{\text {th }} f$ state to be a robust property of TmS and TmSe compounds: it happens irrespective of the precise value of $U_{\text {eff }}$. The degree of occupation and position of the partly occupied $13^{\text {th }} f$ level with respect to the Fermi level is different in TmS and TmSe. In TmS the hole level is almost empty and situated significantly far from the Fermi level. Such a situation is appropriate for achieving the Kondo lattice scenario. In TmSe, the upper hole $13^{\text {th }} f$ level is pinned at the Fermi level. Therefore, TmSe is expected to be an intermediate valent compound in agreement with experimental data.

The LSDA $+U$ approach to band structure calculations of $\mathrm{Yb}_{4} \mathrm{As}_{3}$ produce a band gap between the As $p$ band and the $\mathrm{Yb} 5 d$ band. As a consequence, a very narrow marginally occupied $\mathrm{Yb}^{3+} 4 f$ hole band is pinned close to the top of the As $p$ valence band via the charge balance between $\mathrm{Yb}$ and As which is governed by the large $(\approx 30 \mathrm{eV})$ bare Coulomb integral of the $\mathrm{Yb} 4 f$ state. The same charge balance pins the Fermi level close to the bottom of this $4 f$ hole band which has a width of $0.007 \mathrm{eV}(80 \mathrm{~K})$. It leads to an extremely low carrier density in $\mathrm{Yb}_{4} \mathrm{As}_{3}$. So, for $\mathrm{Yb}_{4} \mathrm{As}_{3}$, the LSDA $+U$ band structure calculations provide a two-band model of the electronic structure: (1) a wide, nearly fully occupied As $4 p$ valence band and (2) a very narrow, marginally occupied $\mathrm{Yb} 4 f$ band, very weakly hybridized with the As $4 p$ band in a small $k$ space region near the $\Gamma$ point of the BZ. Pressure, doping, etc. may slightly change the relative position of these two bands and hence strongly affect the low carrier density and the transport properties. But the $4 f$ shell of the $\mathrm{Yb}^{3+}$ ions is little effected as long as the charge order is maintained.

We would like to point out that the LSDA $+U$ method which combines LSDA with a basically static, i.e., Hartree-Fock-like, mean-field approximation for a multiband Anderson lattice model does not contain true many-body physics. However, this method can be considered as the first step towards a better description of strongly correlated electron systems. The LSDA $+U$ method gave a correct insulating ground state solution for Sm monochalcogenides at ambient pressure. This method provides the correct energy positions of $4 f$ energy bands and gives a reasonable description of the optical properties in $\operatorname{SmX}\left(X=S\right.$, Se, Te) and $\mathrm{SmB}_{6}$. However, the paramagnetic high pressure golden phase of $\mathrm{SmS}$ as well as the formation of the MV state in $\mathrm{SmB}_{6}$ clearly requires a treatment that goes beyond a static mean-field approximation and includes dynamical effects, e.g., the frequency dependence of the self-energy. 


\section{References}

1. Nemoshkalenko V.V., Antonov V.N. Computational Methods in Solid State Physics. Amsterdam, Gordon and Breach Science Publication, 1998.

2. Antonov V.N., Harmon B.N., Yaresko A.N. Electronic Structure and MagnetoOptical Properties of Solids. Amsterdam, Kluwer, 2004.

3. Anisimov V.I., Zaanen J., Andersen O.K. // Phys. Rev. B, 1991, vol. 44, p. 943.

4. Yaresko A.N., Antonov V.N., Fulde P. // Phys. Rev. B, 2003, vol. 67, p. 155103.

5. Anisimov V.I., Aryasetiawan F., Lichtenstein A.I. // J. Phys.: Condens. Matter, 1997, vol. 9, p. 767.

6. Hedin L., Lundqvist B.I. // J. Phys. C, 1971, vol. 4, p. 2064-2084.

7. Antonov V.N., Harmon B.N., Yaresko A.N., Perlov A.Y. // Phys. Rev. B, 1999, vol. 59, p. 14571-14582.

8. Antonov V.N., Harmon B.N., Yaresko A.N. // Phys. Rev. B, 2001, vol. 63, p. 205112.

9. Andersen O.K. // Phys. Rev. B, 1975, vol. 12, p. 3060.

10. Nemoshkalenko V.V., Krasovskii A.E., Antonov V.N., Antonov Vl.N., Fleck U., Wonn H., Ziesche P. // Phys. Status Solidi B, 1983, vol. 120, p. 283-296.

11. von Barth U., Hedin L. // J. Phys. C, 1972, vol. 5, p. 1629.

12. Blöchl P.E., Jepsen O., Andersen O.K. // Phys. Rev. B, 1994, vol. 49, p. 16.

13. Antonov V.N., Bagljuk A.I., Perlov A.Y., Nemoshkalenko V.V., Antonov Vl.N., Andersen O.K., Jepsen O. // Low Temp. Phys., 1993, vol. 19, p. 494.

14. Antonov V.N., Yaresko A.N., Perlov A.Y., Thalmeier P., Fulde P., Oppeneer P.M., Eschrig H. // Phys. Rev. B, 1998, vol. 58, p. 9752-9762.

15. Antonov V.N., Harmon B.N., Perlov A.Y., Yaresko A.N. // Phys. Rev. B, 1999, vol. 59 , p. 14561.

16. Antonov V.N., Harmon B.N., Yaresko A.N. // Phys. Rev. B, 2002, vol. 66, p. 165208.

17. Antonov V.N., Harmon B.N., Yaresko A.N. // Phys. Rev. B, 2002, vol. 66, p. 165209.

18. Harmon B.N., Antonov V.N. // J. Appl. Phys., 2002, vol. 91, p. 9815-9820.

19. Harmon B.N., Antonov V.N. // J. Appl. Phys., 2003, vol. 93, p. 4678-4685.

20. Yaresko A.N., Antonov V.N., Eschrig H., Thalmeier P., Fulde P. // Phys. Rev. B, 2000, vol. 62, p. 15538-15546.

21. Atzkern S., Knupfer M., Golden M.S., Fink J., Yaresko A.N., Antonov V.N., Hübsch A., Waidacher C., Becker K.W. // Phys. Rev. B, 2001, vol. 63, p. 165113.

22. Antonov V.N., Harmon B.N., Antrpov V.P., Perlov A.Y., Yaresko A.N. // Phys. Rev. B, 2001, vol. 64, p. 134410.

23. Antonov V.N., Harmon B.N., Yaresko A.N. // Phys. Rev. B, 2003, vol. 67, p. 024417.

24. Antonov V.N., Perlov A.Y., Oppeneer P.M., Yaresko A.N., Halilov S.V.

// Phys. Rev. Lett., 1996, vol. 77, p. 5253.

25. Oppeneer P.M., Yaresko A.N., Perlov A.Y., Antonov V.N., Eschrig H.

// Phys. Rev. B, 1996, vol. 54, p. R3706.

26. Yaresko A.N., Oppeneer P.M., Antonov V.N., Perlov A.Y., Eschrig H. // Physica B, 1997, vol. 230-232, p. 513.

27. Perlov A.Y., Oppeneer P.M., Antonov V.N., Yaresko A.N., Yavorsky B.Y.

// J. Appl. Cryst., 1998, vol. 271-274, p. 486.

28. Oppeneer P.M., Perlov A.Y., Antonov V.N., Yaresko A.N., Kraft T., Brooks M.S.

// J. Alloys Comp., 1998, vol. 271-273, p. 831.

29. Antonov V.N., Galli M., Marabelli F., Yaresko A.N., Perlov A.Y., Bauer E. 
// Phys. Rev. B, 2000, vol. 62, p. 1742.

30. Yaresko A.N., Antonov V.N., Harmon B.N. // Phys. Rev. B, 2003, vol. 68, p. 214426.

31. Bucher E., Andres K., diSalvo F.J., Maita J.P., Gossard A.C., Cooper A.S., Hull G.W. // Phys. Rev. B, 1975, vol. 11, p. 500-513.

32. Suryanarayanan R., Güntherodt G., Freeouf J.L., Holzberg F. // Phys. Rev. B, 1975, vol. 12 , p. 4215.

33. Wachter P. Handbook of the Physics and Chemistry of Rare Earths (ed. by Gschneidner K.A., Eyring L., Hüfner S.), vol. 19, p. 177. Amsterdam, North-Holland, 1994.

34. Lassailly Y., Vettier C., Holzberg F., Flouquet J., Zeyen C., Lapierre F.

// Phys. Rev. B, 1983, vol. 28, p. 2880.

35. Berger A., Bucher E., Haen P., Holzberg F., Lapierre F., Penney T., Tournier R. Valence Instabilities and Related Narrow Band Phenomena (ed. by Parks R.D.), p. 491. New York, Plenum, 1977.

36. Haen P., Bioud H., Lapierre F., Holzberg F. - In: Theoretical and Experimental Aspects of Valence Fluctuations, Proceedings of the 5th International Conference on Valence Fluctuations, Bangalore, 1987, (ed. by Gupta L.C., Malik S.K.), p. 445, Plenum, New York.

37. Lapierre F., Haen P., Coqblin B., Ribault M., Holzberg F. // Physica (Amsterdam), 1981, vol. 108B, p. 1351.

38. Lawrence J.M., Riseborough P.S., Parks R.D. // Rep. Prog. Phys., 1981, vol. 44, p. 1.

39. Campagna M., Wertheim G.K., Bucher E. Structure and Bonding, vol. 30. Berlin, Springer, 1976.

40. Campagna M., Bucher E., Wertheim G.K., Buchanan D.N.E., Longinotti L.D. // Phys. Rev. Lett., 1974, vol. 32, p. 885.

41. Kaindl G., Laubschat C., Reihl B., Pollak R.A., Martensson N., Holzberg F., Eastman D.E. // Phys. Rev. B, 1982, vol. 26, p. 1713.

42. Wertheim G.K., Eib W., Kaldis E., Kampagna M. // Phys. Rev. B, 1980, vol. 22, p. 6240 .

43. Ufuktepe Y., Kimura S., Kinoshita T., Nath K.G., Kumigashira H., Takahashi T., Matsumura T., Suzuki T., Ogasawara H., Kotani A. // J. Phys. Soc. Jpn., 1998, vol. 67, p. 2018.

44. Batlogg B., Ott H.R., Kaldis E., Thoni W., Wachter P. // Phys. Rev. B, 1979, vol. 19, p. 247.

45. Haen P., Lapierre F., Mignot J.M., Tournier R., Holzberg F. // Phys. Rev. Lett., 1979, vol. 43, p. 304.

46. Ribault M., Haen J. F.P., Lapierre F., Mignot J.M., Holzberg F. // Phys. Rev. Lett., 1980, vol. 45, p. 1295.

47. Andres K., Walsh W.M., Jr, Darack S., Rupp L.W., Jr, Longinotti L.D.

// Solid State Commun., 1978, vol. 27, p. 825.

48. Berton A., Chaussy J., Cornut B., Flouquet J., J.Odin, Peyrard J., Holzberg F.

// Phys. Rev. B, 1981, vol. 23, p. 3504.

49. Holzberg F., Penney T., Tournier R. // J. Phys. (Paris), 1979, vol. 40, No. C5, p. 314.

50. Kaldis E., Frizler B. // Prog. Solid State Chem., 1982, vol. 14, p. 95.

51. Hayen P., Holzberg F., Lapierre F., Penney T., Tournier R. Valence Instabilities and Related Narrow Band Phenomena (ed. by Parks R.D.), p. 495. New York, Plenum, 1977. 
52. Lassailly Y., Vettier C., Holzberg F., Benoit A., Flouquet J. // Solid State Commun., 1984, vol. 52, p. 717 .

53. Clementyev E., Köhler R., Braden M., Mignot J.-M., Vettier C., Matsumura T., Suzuki T. // Physica B, 1977, vol. 230-232, p. 735.

54. Yeh J.J., Lindau I. // Atomic data and Nuclear data tables, 1985, vol. 32, p. 1.

55. Wachter P., Kamba S., Grioni M. // Physica B, 1998, vol. 252, p. 178.

56. Cox P.A. Structure and Bonding, vol. 24. Berlin-Heidenberg-New York, Springer, 1975.

57. Racah G. // Phys. Rev., 1949, vol. 76, p. 1352.

58. Batlogg B. // Phys. Rev. B, 1981, vol. 23, p. 1827.

59. Hirst L.L. // Phys. Condens. Matter., 1970, vol. 11, p. 255.

60. Varma C.M. // Rev. Mod. Phys., 1976, vol. 48, p. 219.

61. Marabelli F., Wachter P. Theoretical and Experimental Aspects of Valence Fluctuations and Heavy Fermions (ed. by Gupta L.C., Malik S.K.), p. 269. New York, Plenum Press, 1987.

62. Campagna M., Bucher E., Wertheim G.K., Longinotti L.D. // Phys. Rev. Lett., 1974, vol. 33, p. 165.

63. Batlogg B., Kaldis E., Schlegel A., Wachter P. // Phys. Rev. B, 1976, vol. 14, p. 5503.

64. Jayaraman A.V., Narayanamurti V., Bucher E., Maines R.G. // Phys. Rev. Lett., 1970, vol. 25, p. 368.

65. Bucher E., Narayanamurti V., Jayaraman A.V. // J. Appl. Phys., 1971, vol. 42, p. 1741.

66. Sovestnov A.F., Shaburov V.A., Markova L.A., Savitski E.M. // Fiz. Tverd. Tela (Leningrad), 1981, vol. 23, p. 2827. [Sov. Phys. Solid State, 1981, vol. 23, p. 1652].

67. Coey J. M.D., Glatak S.K., Avignon M., Holtzberg P. // Phys. Rev. B, 1976, vol. 14, p. 3744.

68. Maple M.B., Wohleben D. // Phys. Rev. Lett., 1976, vol. 14, p. 3744.

69. Travaglini G., Wachter P. // Phys. Rev. B, 1984, vol. 30, p. 5877.

70. Bader S.D., Phillips N.E., McWhan D.B. // Phys. Rev. B, 1973, vol. 7, p. 4686.

71. Vanstein E.E., Blokhin S.M., Paderno Y.B. // Sov. Phys. Solid State, 1965, vol. 6, p. 281.

72. Riseborough P.S. // Advances in Physics, 2000, vol. 49, p. 257.

73. Degiorgi L. // Rev. Mod. Phys., 1999, vol. 71, p. 687.

74. Fisk Z., Sarrao J.L., Cooper S.L., Nyhus P., Boehinger G.S., Passner A., Canfield P.C. // Physica B, 1996, vol. 223-224, p. 409.

75. von Molnar S., Theis T., Benoit A., Briggs A., Floquet J., Ravex J., Fisk Z. Valence Instabilities (ed. by Wachter P., Boppart H.), p. 389. Amsterdam, North-Holland, 1982.

76. Nanba T., Ohta H., Motokowa M., Kimura S., Kunil S., Kasuya T. // Physica B, 1993, vol. 186-188, p. 440.

77. Alekseev P.A., Lazukov V.A., Osborn R., Rainford B.D., Sadikov I.P., Konovalova E.S., Paderno Y.B. // Europhys. Lett., 1993, vol. 23, p. 347.

78. Mignot J.-M., Alekseev P.A., Rossat-Mignot J., Lazukov V.N., Sadikov I.P. // Physica B, 1994, vol. 199-200, p. 430.

79. Alekseev P.A., Mignot J.-M., Rossat-Mignot J., Lazukov V.N., Sadikov I.P., Konovalova E.S., Paderno Y.B. // J. Phys.: Condens. Matter, 1995, vol. 7, p. 289.

80. Mignot J.-M., Alekseev P.A. // Physica B, 1995, vol. 215, p.99. 
81. Travaglini G., Wachter P. // Phys. Rev. B, 1984, vol. 29, p. 893.

82. Battlog B., Schmidt P.H., Rowwel J.M. Valence Fluctuations in Solids (ed. by Falikov L.M., Hunke W.), p.357. Amsterdam, North-Holland M.B.M., 1981.; Frankowski L., Wachter P. // Solid State Commun., 1982, vol. 41, p. 577.

83. Menth A., Buetler E., Geballe T.H. // Phys. Rev. Lett., 1969, vol. 22, p. 295.

84. Nickerson J.C., White R.M., Lee K.N., Bachmann R., Geballe T.H., Hull G.W. // Phys. Rev. B, 1971, vol. 3, p. 2030.

85. Allen J.W., Battlog B., Wachter P. // Phys. Rev. B, 1979, vol. 20, p. 4807.

86. Cooley J.C., Aronson M.C., Fisk Z., Canfield P.C. // Phys. Rev. Lett., 1995, vol. 74, p. 1629.

87. Kasuya T., Takegahara K., Fujita T., Tanaka T., Bannai E. // J. Phys. (Paris) Colloq., 1979, vol. 40, p. C5-308.

88. Morillo J., Bordier G., deNovion C.H., Senateur J.P., Jun J. // J. Magn. Magn. Mater., 1985, vol. 47-48, p. 465.

89. Geballe T.H., Menth A., Buehler E., Hall G.W. // J. Appl. Phys., 1979, vol. 41, p. 904.

90. Koni S. // J. Magn. Magn. Mater., 1987, vol. 63-64, p. 673.

91. Gorshunov B., Sluchanko N., Volkov A., Dressel M., Krebel G., Loidl A., Konii S. // Phys. Rev. B, 1999, vol. 59, p. 1808.

92. Taraskon J.M., Isikawa Y., Chevalier B., Etourneau J., Hagenmuller P., Kasuya M. // J. Physique, 1980, vol. 41, p. 1141.

93. Kasuya T., Kasaya M., Takegahara K., Fujita T., Goto T., Tamaki A., Takigawa M., Yasuoka H. // J. Magn. Magn. Mater., 1983, vol. 31-34, p. 447.

94. Cohen R.L., Eibschutz M., West K.W., Buller E. // J. Appl. Phys., 1970, vol. 41, p. 898.

95. Yanase A., Harima H. // Progr. Theor. Phys. Suppl., 1992, vol. 108, p. 19.

96. Kierzek-Pecold E. // Phys. Status Solidi, 1969, vol. 33, p. 523.

97. Kimura S., Nanba T., Kunii S., Kasuya T. // J. Phys. Soc. Jpn., 1990, vol. 59, p. 3388 .

98. Kimura S., Nanba T., Tomikawa T., Kunii S., Kasuya T. // Phys. Rev. B, 1992, vol. 46, p. 12196.

99. Kimura S., Nanba T., Kunii S., Kasuya T. // Phys. Rev. B, 1994, vol. 50, p. 1406.

100. Ochiai A., Suzuki T., Kasuya T. // J. Phys. Soc. Jpn., 1990, vol. 59, p. 4129.

101. Ochiai A., Aoki H., Suzuki T., Helfrich R., Steglich F. // Physica B, 1997, vol. 230232, p. 708.

102. Bonville P., Ochiai A., Suzuki T., Vincent E. // J. Phys. I France, 1994, vol. 4, p. 595.

103. Reinders P.H.P., Ahlheim U., Fraas K., Steglich F., Suzuki T. // Physica B, 1993, vol. 186-188, p. 434.

104. Suzuki T. // Physica B, 1993, vol. 186-188, p. 347.

105. Suzuki T. // Jap. J. Appl. Phys., 1993, vol. 8, p. 267.

106. Ochiai A., Li D.X., Haga Y., Nakamura O., Suzuki T. // Physica B, 1993, vol. 186188, p. 437.

107. Kasuya T. // J. Phys. Soc. Jpn., 1994, vol. 63, p. 2481.

108. Suzuki T. // Jap. J. Appl. Phys., 1993, vol. 8, p. 44.

109. O'Keeffe M., Andersson S. // Acta Crystallogr. A, 1977, vol. 33, p. 914.

110. Kohgi M., Iwasa K., Ochiai A., Suzuki T., Mignot J.-M., Gillon B., Gukasov A., Schweizer J., Kakurai K., Nishi M., Dönni A., Osakabe T. // Physica B, 1997, 
vol. 230-232, p. 638.

111. Goto T., Nemoto Y., Nakamura S., Ochiai A., Suzuki T. // Physica B, 1997, vol. 230232 , p. 702.

112. Suzuki T., Suzuki T., Haga Y., Li D.X., Matsumura T., Hotta E., Uesawa A., Kohgi M., Osakabe T., Takagi S., Suzuki H. // Physica B, 1995, vol. 206-207, p. 771.

113. Okunuki Y., Mori N., Ochiai A., Haga Y., Suzuki T. // J. Phys. Soc. Jpn., 1995, vol. 64 , p. 533.

114. Mori N., Takahashi H., Sekine Y., Ochiai A., Haga Y., Suzuki T., Kashiwakura T., Nakai S., Nomura M. // Physica B, 1997, vol. 230-232, p. 630.

115. Suga S., Ogawa S., Namatame H., Taniguchi M., Kakizaki A., Ishii T., Fujimori A., Oh S.-J., Kato H., Miyahara T., Ochiai A., Suzuki T., Kasuya T. // J. Phys. Soc. Jpn., 1989, vol. 58, p. 4534.

116. Saitoh Y., Suga S., Matsubara H., Tsukikawa Y., Mori Y., Oyamada A., Ochiai A., Suzuki T., Kasuya T. // J. Phys. Soc. Jpn., 1991, vol. 60, p. 4005.

117. Kimura S., Ikezawa M., Ochiai A., Suzuki T. // J. Phys. Soc. Jpn., 1996, vol. 65, p. 3591.

118. Kimura S., Ochiai A., Suzuki T. // Physica B, 1997, vol. 230-232, p. 705.

119. Kwon Y.S., Ochiai A., Kitazawa H., Sato N., Abe H., Nanba T., Ikezawa M., Takegahara K., Suzuki T., Kasuya T. // J. Magn. Magn. Mater., 1987, vol. 70, p. 397.

120. Kasuya T. // J. Phys. Soc. Jpn., 1994, vol. 63, p. 843.

121. Fulde P., Schmidt B., Thalmeier P. // Europhys. Lett., 1995, vol. 31, p. 323.

122. Schmidt B., Thalmeier P., Fulde P. // Physica B, 1996, vol. 223-224, p. 373.

123. Li Y.M., d'Ambrumenil N., Fulde P. // Phys. Rev. Lett., 1997, vol. 78, p. 3386.

124. Takegahara K., Kaneta Y. // J. Phys. Soc. Jpn., 1991, vol. 60, p. 4009.

125. Fulde P. Electron Correlations in Molecules and Solids. Berlin, Springer, 1995.

Електронна структура змішаних валентних систем.

\author{
В.М.Антонов ${ }^{1}$, А.П.Шпак ${ }^{1}$, А.М.Яресько ${ }^{2}$ \\ 1 Інститут Металофізики Національної академії наук України, \\ бульв. Вернадського 36, 03142 Київ \\ 2 Інститут Макса Планка Комплексних систем, \\ D-01187 Дрезден, Німеччина
}

Отримано 24 березня 2004 р.

Електронна структура монохалкогенідів Tm i Sm, SmB6 і Yb4As3 теоретично досліджується із перших принципів, використовуючи повністю релятивістський діраківський ЛМТО метод зонних структур. Електронна структура отримана, використовуючи наближення локальної спінової густини (LSDA), наряду з так званим LSDA+U підходом. В рамках звичайного LSDA не вдається коректно описати електронну структуру таких матеріалів завдяки сильному Кулонівському відштовхуванню на вузлі, тоді як LSDA+U підхід чудово точний при наданні детальної згоди з експериментом для ряду властивостей.

Ключові слова: сильно корельовані системи, оптика, зонна структура

PACS: $71.28 .+d$, 71.25.Pi, 75.30.Mb 